\title{
SPITZER INFRARED SPECTROGRAPH SPECTROSCOPY OF THE 10 Myr OLD EF Cha DEBRIS DISK: EVIDENCE FOR PHYLLOSILICATE-RICH DUST IN THE TERRESTRIAL ZONE
}

\author{
Thayne Currie ${ }^{1}$, Carey M. Lisse ${ }^{2}$, Aurora Sicilia-Aguilar ${ }^{3}$, George H. Rieke ${ }^{4}$, And Kate Y. L. Su ${ }^{4}$ \\ ${ }^{1}$ NASA-Goddard Space Flight Center, Greenbelt, MD 20771, USA \\ 2 Johns Hopkins University Applied Physics Laboratory, 11100 Johns Hopkins Road, Laurel, MD 20723-6099, USA \\ ${ }^{3}$ Max-Planck-Institute for Astronomy, Königstuhl 17, 69117 Heidelberg, Germany \\ ${ }^{4}$ Steward Observatory, University of Arizona, 933 North Cherry Avenue, Rm. N204, Tucson, AZ 85721-0065, USA \\ Received 2010 July 7; accepted 2011 April 6; published 2011 June 6
}

\begin{abstract}
We describe Spitzer Infrared Spectrograph spectroscopic observations of the $\sim 10$ Myr old star, EF Cha. Compositional modeling of the spectra from $5 \mu \mathrm{m}$ to $35 \mu \mathrm{m}$ confirms that it is surrounded by a luminous debris disk with $L_{D} / L_{\star} \sim 10^{-3}$, containing dust with temperatures between $225 \mathrm{~K}$ and $430 \mathrm{~K}$, characteristic of the terrestrial zone. The EF Cha spectrum shows evidence for many solid-state features, unlike most cold, low-luminosity debris disks but like some other 10-20 Myr old luminous, warm debris disks (e.g., HD 113766A). The EF Cha debris disk is unusually rich in a species or combination of species whose emissivities resemble that of finely powdered, laboratory-measured phyllosilicate species (talc, saponite, and smectite), which are likely produced by aqueous alteration of primordial anhydrous rocky materials. The dust and, by inference, the parent bodies of the debris also contain abundant amorphous silicates and metal sulfides, and possibly water ice. The dust's total olivine to the pyroxene ratio of $\sim 2$ also provides evidence of aqueous alteration. The large mass volume of grains with sizes comparable to or below the radiation blow-out limit implies that planetesimals may be colliding at a rate high enough to yield the emitting dust but not so high as to devolatize the planetesimals via impact processing. Because phyllosilicates are produced by the interactions between anhydrous rock and warm, reactive water, EF Cha's disk is a likely signpost for water delivery to the terrestrial zone of a young planetary system.
\end{abstract}

Key words: astrochemistry - infrared: stars - planets and satellites: formation - protoplanetary disks - stars: individual (EF Cha) - techniques: spectroscopic

Online-only material: color figures

\section{INTRODUCTION}

The 10-20 Myr age range is an important time in the formation of icy planetesimals and planets around $\sim 2 M_{\odot}$ stars. While debris emission decays with time for $t>20 \mathrm{Myr}$ as expected for simple collisional grinding of a fixed-mass planetesimal belt (Rieke et al. 2005; Wyatt et al. 2007), debris emission for A/F stars is observed to rise from $5 \mathrm{Myr}$ to $10 \mathrm{Myr}$ and peak from 10 to $20 \mathrm{Myr}$ (Currie et al. 2008a; Hernandez et al. 2009; Currie et al. 2008b, 2009). The rise in debris emission has been attributed to the agglomerate accretion of $1000 \mathrm{~km}$-sized icy bodies in outer disk regions as planet formation switches from a fast runaway growth stage to a slower, debris-producing oligarchic stage (Kenyon \& Bromley 2008, 2010). Dynamical perturbations by massive planets may also explain the luminous debris emission at these ages (Mustill \& Wyatt 2009; Kennedy \& Wyatt 2010).

Spitzer has observed a number of luminous debris disks in this critical stage. A wide range of processes appear to be responsible for their extreme infrared emission, potentially revealing a variety of situations prevailing in their terrestrial planet zones. For example, HD 113766A, an F3/5 star in 10-16 Myr old Lower Centaurus Crux, shows evidence for multiple debris belts, including a warm $(T \sim 440 \mathrm{~K})$ silicate-dominated debris disk with a composition consistent with S-type asteroids and whose flux densities require an enormous reservoir $\left(M \gtrsim M_{\text {Mars }}\right)$ of colliding material (Chen et al. 2006; Lisse et al. 2008). Lisse et al. (2008) interpreted these spectral features as evidence of terrestrial planet-forming events. HD 172555, a $\sim 12 \mathrm{Myr}$ old A5 star, also exhibits luminous warm debris disk emission at
$L_{D} / L_{*} \sim 10^{-3}$, but contains spectral features consistent with silica and $\mathrm{SiO}$ gas produced by a giant hypervelocity collision, similar to that likely responsible for forming the present-day Earth-Moon system (Lisse et al. 2009). Eta Corvi, a 1 Gyr old F2 star, shows an extended cold Kuiper Belt disk due to collisions driven by dynamical stirring, and a separate belt of warm inner system dust due to the collision of a Kuiper Belt Object with a planet in the terrestrial habitable zone (Wyatt et al. 2005; Lisse et al. 2011a).

Spatially resolved images of other luminous debris disks at these ages — such as HR 4796A (A0V) and $\beta$ Pic (A5V)—find evidence for debris disk dynamical sculpting, such as asymmetries or warping, plausibly due to the presence of massive planets (Mouillet et al. 1997; Golimowski et al. 2006; Schneider et al. 2009; Lagrange et al. 2010). However, unlike HD 113766A and HD 172555, they have rather featureless IR spectra, lacking evidence for massive collisions (Chen et al. 2006). The disk around 49 Ceti (A0V) extends at high surface brightness to a radius of $900 \mathrm{AU}$ but appears to have been cleared within $20 \mathrm{AU}$ of the star, again possibly due to an unseen planet (Wahhaj et al. 2007). Modeling of the images suggests that the mid-infrared emission from the 49 Ceti disk is dominated by small, short-lived grains ( $\sim 0.01 \mu \mathrm{m}$; Wahhaj et al. 2007).

EF Chameleonis (hereafter EF Cha) provides another interesting example of the variety of disk properties at this stage. It is an $\sim 10$ Myr old A9IV/V star 95 pc from the Sun identified from the IRAS satellite as having infrared excess emission consistent with a luminous debris disk of $L_{D} / L_{\star} \sim 10^{-3}$ (for $\left.L_{\star} / L_{\odot} \sim 9.7\right)$. A combination of Midcourse Space Experiment (MSX), ground-based, and Spitzer data shows that its disk 
contains warm dust at temperatures $\sim 240 \mathrm{~K}$ (Rhee et al. 2007). It is among the relatively rare disks whose emission is dominated by warm dust and, therefore, by material in the terrestrial-planet zone (Rhee et al. 2007; Currie et al. 2007a, 2007b; Morales et al. 2009).

In this paper, we present and analyze high signal-to-noise 5-35 $\mu \mathrm{m}$ spectra of the EF Cha debris disk obtained with Spitzer. Section 2 describes our observations and data reduction. In Section 3, we compare EF Cha to a simple model for blackbody disk emission and confirm that much of its IR excess is most plausibly due to debris emission originating from EF Cha's terrestrial planet-forming region. We then compare the EF Cha spectrum to spectra from other debris disks, protoplanetary disks, comets, and sophisticated mineralogical models in Section 4 to determine the chemical composition of the EF Cha debris disk dust and constrain the likely origin of its material. We conclude by placing the spectral analysis of the EF Cha debris disk in the context of mineralogical studies of the early solar system and discuss processes likely responsible for explaining the EF Cha disk's spectrum.

\section{OBSERVATIONS AND DATA REDUCTION}

EF Cha was observed by the Spitzer Infrared Spectrograph (IRS) on 2008 September 14 and October 12, in staring mode (PID 50150; AORs r25679360, r25678080, and r25678336). The low-resolution spectra covered 5-38 $\mu \mathrm{m}$, using all four lowresolution orders (Short-Low 1 and 2, Long-Low 1 and 2), with exposures consisting of two cycles with a ramp duration of $6 \mathrm{~s}$ each. The high-resolution spectra covered 9.9-19.6 $\mu \mathrm{m}$ in two cycles with a ramp duration of $30 \mathrm{~s}$ each. For the high-resolution spectra only, we obtained separate background observations.

We processed all spectra using the Spectroscopic Modeling Analysis and Reduction Tool (SMART; Higdon et al. 2004). For the low-resolution spectra, we first median-combined all frames for a specific setting (e.g., Short-Low 1) at a given nod position. Then, we subtracted the median-combined frame for one nod position from each frame corresponding to the other nod position, producing sky-subtracted and bad pixel-mitigated focal plane images. We then grouped the frames together by calibration file and extracted spectra from them simultaneously, using the automatic optimal extraction algorithm provided by SMART. Finally, we grouped the extracted spectra for each setting together, masking the bonus order, clipping obvious outlying pixels and others using the SMART "special" clipping algorithm set at a $2.5 \sigma$ threshold, masking pixels lying outside the wavelength range where IRS calibration is reliable (e.g., $\lambda>38 \mu \mathrm{m}$ ), and median-combined all spectra.

For the high-resolution spectra, we first median-combined the science frames and the sky background frames at a given nod position. The appropriate median-combined sky frame was then subtracted from the science frames at each nod position. Using the full aperture algorithm, we extracted each sky-subtracted frame. The spectra were then grouped together; obvious outlying pixels are identified by comparing pixel values from the four separate exposures, masked, and removed. Each spectrum was defringed with the algorithm irsfringe ported into SMART and trimmed of pixels with unreliable flux calibration as determined from Table 5.1 in the IRS data handbook. As with the lowresolution spectra, we clipped the processed spectra at $2.5 \sigma$ and median-combined them to obtain the final high-resolution spectrum.

Figure 1 (top panel) shows the extracted spectra as a function of wavelength. The low-resolution spectrum (black line) appears to be smooth and high quality from 5 through $\sim 30-35 \mu \mathrm{m}$. As expected, the high-resolution spectrum (gray line, binned by two wavelength elements) is noisier yet faithfully tracks the spectral energy distribution (SED) of the low-resolution spectrum. According to SMART, the low spectra achieve a signal-to-noise ratio of $\sim 100-300$ from 9 through $15 \mu \mathrm{m}$ and above $5 \sigma-10 \sigma$ at all wavelengths (bottom panel). The signalto-noise ratio for the high-resolution spectrum ranges from $\sim 50$ at $10 \mu \mathrm{m}$ to $\sim 10$ at $19 \mu \mathrm{m}$.

To provide a check on the signal to noise and reliability of our low-resolution spectrum, we performed two tests. First, we extracted spectra from the two nod positions separately and then compared the flux densities at each wavelength (Figure 2). Through $15 \mu \mathrm{m}$ the spectra show very good agreement. The spectra become noisier at $15-30 \mu \mathrm{m}$ and then produce highly uncertain fluxes at $\sim 30-38 \mu \mathrm{m}$. These comparisons allow us to empirically estimate the signal to noise per nod position as a function of wavelength (right panel). Through $15 \mu \mathrm{m}$ the median signal to noise is $\sim 57$ per nod. From $15 \mu \mathrm{m}$ to $30 \mu \mathrm{m}$, the median signal to noise per nod is $\sim 37$. The median signal to noise at $30-38 \mu \mathrm{m}$ is 6.4 . The median signal to noise of the combined and extracted spectrum should then be $\sim 81$ through $15 \mu \mathrm{m}, \sim 52$ from $15-30 \mu \mathrm{m}$, and $\sim 9$ at $30-38 \mu \mathrm{m}$. While these estimates are about a factor of two lower than those for the combined spectrum as derived by SMART, they confirm that the spectrum has a high signal to noise through $\sim 30 \mu \mathrm{m}$.

Second, we compared our SMART spectral extraction to that from the Formation and Evolution of Planetary Systems (FEPS) pipeline (Bouwman et al. 2008). Figure 3 displays the two extractions. The FEPS pipeline-produced spectrum consistently has a higher flux than the SMART extraction (top panel). However, rescaling the FEPS spectrum by 0.95 yields an almost perfect agreement between the two extractions (bottom panel). Since the FEPS and SMART-produced spectra are essentially scaled versions of one another, the differences likely arise from the way in which pointing offset refinements are treated, which compensate for the target not being precisely positioned at the center of the slit (J. Bouwman 2010, private communication; Swain et al. 2008). We conclude that our spectral extraction method is robust and interpreting the presence of spectral features, which depend on the relative flux at different wavelengths, is not hindered by uncertainties/errors in the absolute flux calibration from our extraction method.

In part, these tests were performed to interpret strong features near $\sim 10 \mu \mathrm{m}$. The IRS SL1 order has a permanent bad pixel lying very close to the spectrum at one of the nod positions near $\sim 10 \mu \mathrm{m}$ : failure to properly identify and mitigate this pixel can lead to a spurious strong emission peak (G. Bryden 2009, private communication). Given the excellent agreement between spectra extracted from different nod positions and that extracted using the FEPS pipeline, we conclude that we have successfully corrected for any such bad pixel-induced spectral artifacts.

\section{ANALYSIS}

\subsection{Basic Properties of the EF Cha Disk: Disk Evolutionary State and Dust Temperature}

First, we examine the spectra, looking for any evidence of nebular gas emission expected if the EF Cha disk is a "remnant" protoplanetary disk instead of a debris disk. Neither the low nor the high-resolution spectra identify evidence for $\mathrm{H}_{2}$ gas emission at $12.1 \mu \mathrm{m}, 17 \mu \mathrm{m}$, or $28 \mu \mathrm{m}$ expected for disks with copious amounts of gas (e.g., Gorti \& Hollenbach 2004; Pascucci et al. 

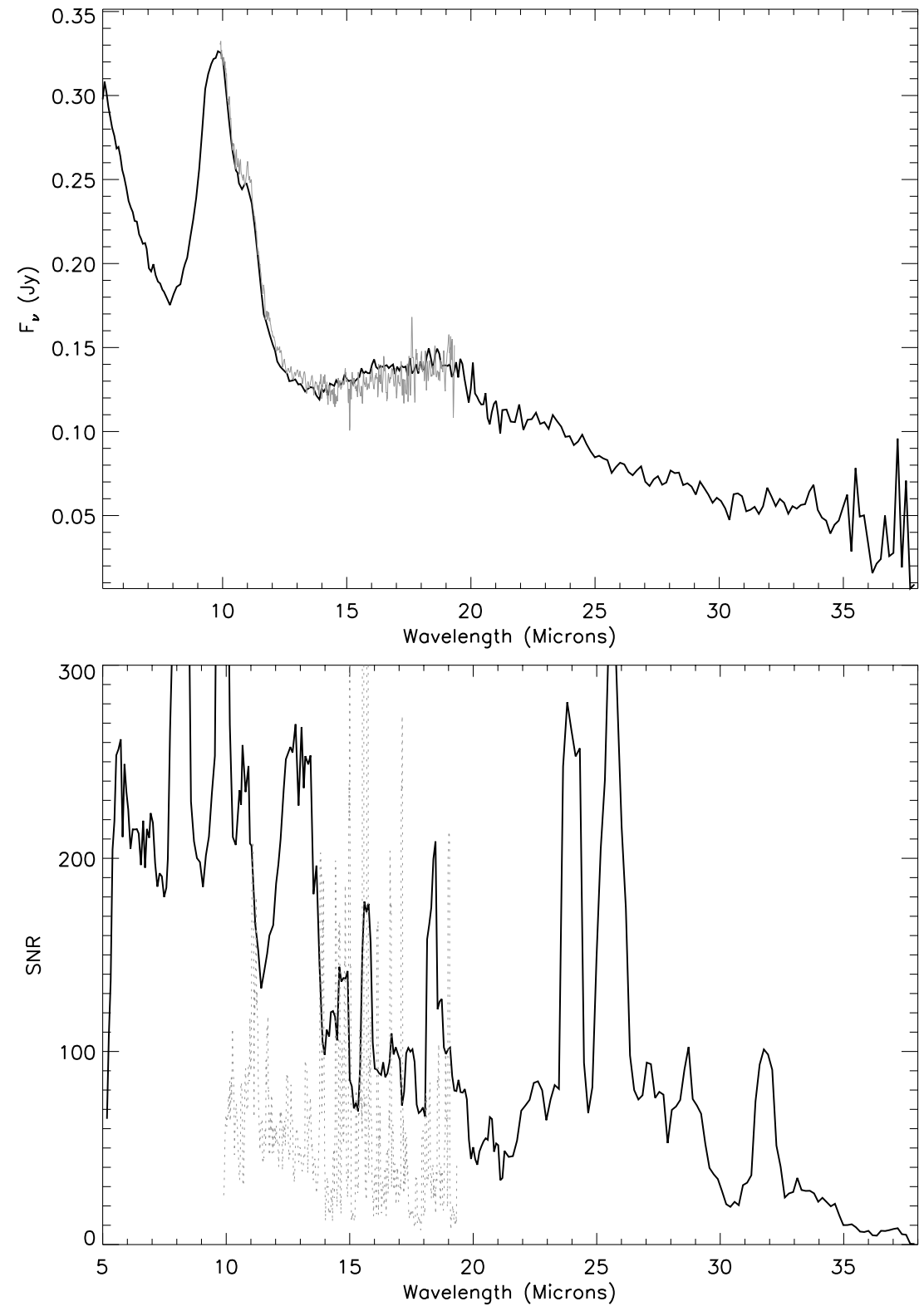

Figure 1. Top panel: Spitzer IRS low-resolution (black line) and high-resolution (gray line) spectra of EF Cha. Bottom panel: signal-to-noise of the low-resolution and high-resolution spectra vs. wavelength. The plotted signal to noise is smoothed by five pixels in wavelength.
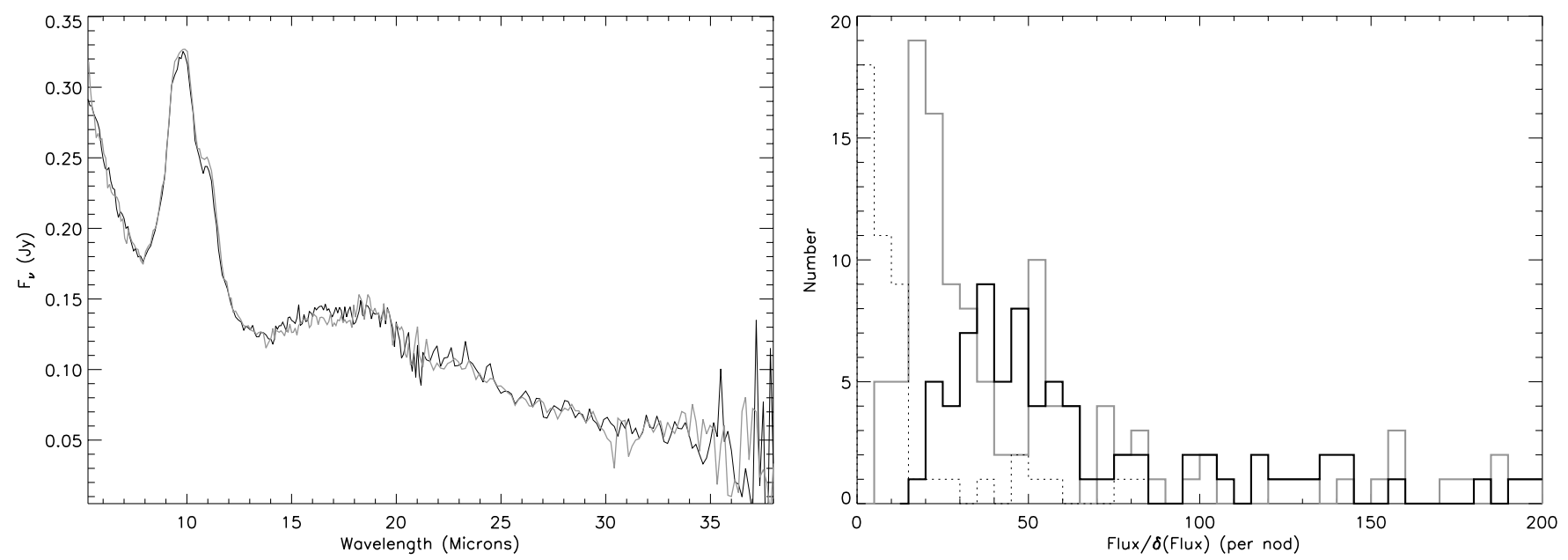

Figure 2. Left: extracted low-resolution spectra for each of the two nod positions (black and gray lines). Right: histogram plot of the flux of the first nod position divided by the flux difference between the nod positions. The distributions are separated by wavelength: 6-15 $\mu \mathrm{m}$ (black line), 15-30 $\mu \mathrm{m}$ (gray line), and 30-38 $\mu \mathrm{m}$ (dotted line). 

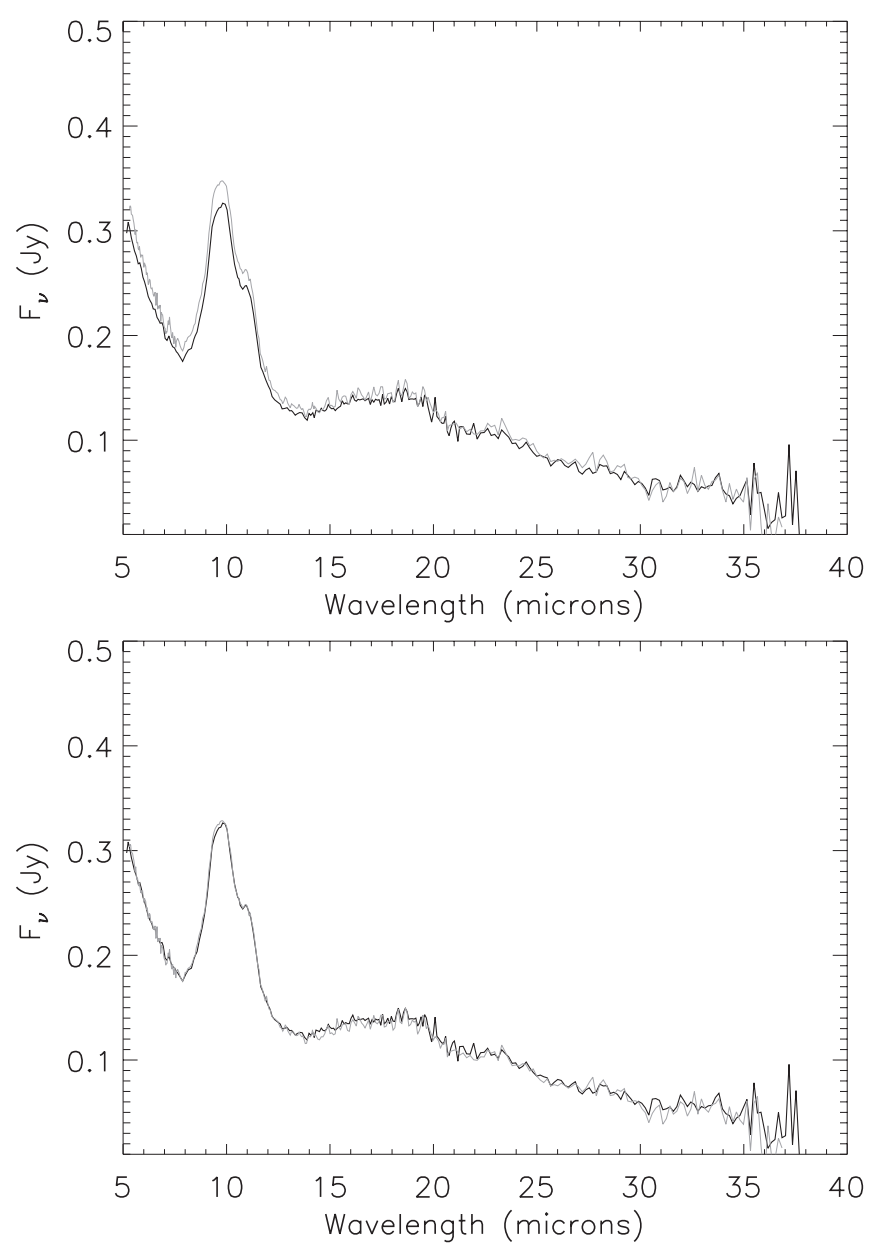

Figure 3. Comparison between the EF Cha IRS spectral extraction produced from SMART and that produced from the FEPS pipeline (top panel). The same comparison with the FEPS spectrum rescaled by 0.95, a $5 \%$ decrease (Bottom panel).

2006; Carmona et al. 2008; Martin-Zaidi et al. 2010). Neither do we find evidence for [Ne II] emission at $\sim 12.8 \mu \mathrm{m}$, indicative of trace amounts $\left(\sim 10^{-6} M_{J}\right)$ of circumstellar gas (Pascucci et al. 2007). There may be a very faint, low signal-to-noise emission feature at $\sim 6 \mu \mathrm{m}$ corresponding to water gas, though its significance is less than $3 \sigma$. Since this emission, if real, can be produced by evaporating icy bodies it does not imply the presence of substantial primordial nebular gas.

Detecting gas emission lines like $\mathrm{H}_{2}$ and [Ne II] has proved difficult for younger stars with masses like EF Cha's (e.g., Juhasz et al. 2010), some of which like AB Aur have direct evidence for circumstellar gas (Bitner et al. 2007). Because clear mid-IR detections of gas may only be possible for small fractions of the Herbig AeBe disk lifetime (e.g., Martin-Zaidi et al. 2010), the absence of these lines does not guarantee the absence of gas in the system. However, less than $\sim 1 \%-2 \%$ of stars as old as EF Cha show evidence for gas (e.g., Currie et al. 2007c), and most protoplanetary disks around $M \approx 2 M_{\odot}$ stars disappear prior to 3 Myr (Currie \& Kenyon 2009). The EF Cha disk is also optically thin at all wavelengths (Rhee et al. 2007) and its emission can be well fit with one or two single temperature blackbodies consistent with narrow debris belts (Rhee et al. 2007; Sections 3.2 and 3.3 below). Protoplanetary disks, on the other hand, are typically optically thick in the mid-IR and are better fit by disks with large spatial extents. Both the lowresolution and high-resolution spectra contain a strong, broad

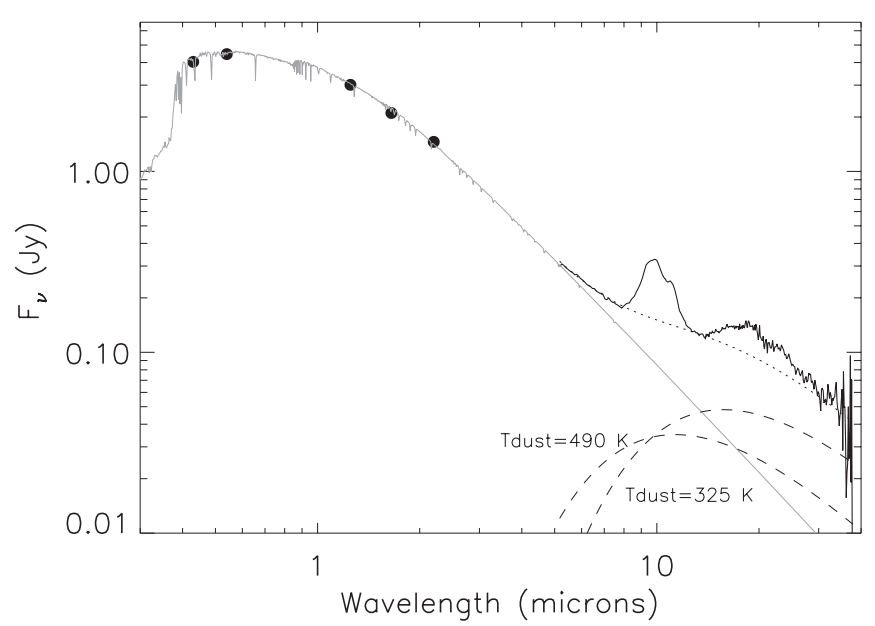

Figure 4. EF Cha SED including photometric data from the Tycho-II survey and 2MASS (dots) and IRS data (solid black line). The dashed lines correspond to the star + single-temperature blackbodies; the dotted line corresponds to the combined flux from these blackbodies+the star's flux. For the star's flux, we fit the SED to a Kurucz stellar atmosphere model for a $T_{e} \sim 7400 \mathrm{~K}$ star (gray line).

$10 \mu \mathrm{m}$ silicate emission feature that is due to the presence of many small, micron-sized dust grains. Thus, to first order the mid-IR spectrum of EF Cha is consistent with that expected for a gas-poor, dusty debris disk, as argued by Rhee et al. (2007).

Next, we construct a full SED of the EF Cha system to determine the temperature of the dust producing the observed IRS excess emission. To our Spitzer data, we add B and V data from the Tycho-II catalog and $J H K_{s}$ data from the Two Micron All Sky Survey (2MASS; Skrutskie et al. 2006; Figure 4). We fit the optical data to the appropriate Kurucz stellar atmosphere model for an A9 star, using the effective temperature scale from Currie et al. (2010). Removing the stellar photosphere model from the total IRS spectrum, we find disk excess emission rising above the noise level at $\sim 5-6 \mu \mathrm{m}$ and dominating the system emission by $10 \mu \mathrm{m}$.

To make an initial determination of the typical dust temperature, we fit the observed SED to a simple two-temperature blackbody model, with dust temperature ranges of $50-400 \mathrm{~K}$ for the cool component and 300-1000 K for the hot component. In doing this, we required that the model reproduce the observed SED in regions lacking obvious solid-state features ( $\lambda \sim 9-12 \mu \mathrm{m}, 15-25 \mu \mathrm{m}$ ). The SED is best modeled by dust belts with temperatures of $\sim 325 \mathrm{~K}$ and $490 \mathrm{~K}$, corresponding to dust in the terrestrial zone of the system. More generally, the SED at $30-35 \mu \mathrm{m}$ is consistent with an $F_{\nu} \propto \lambda^{-2}$ decline expected for warm dust in the Rayleigh-Jeans limit. Assuming a luminosity of $9.7 L_{\odot}$ for $\mathrm{EF}$ Cha and the blackbody relations between dust temperature and location- $T_{\text {dust }} \sim 280 \mathrm{~K} \times$ $(r / 1 \mathrm{AU})^{-1 / 2}\left(L_{\text {star }} / L_{\text {Sun }}\right)^{1 / 4}$ these temperatures imply the presence of dust belts at 1.0 AU and 2.4 AU. As we were able to fit the entirety of the observed flux to within $20 \%$, we conclude, based on these simple fits, that EF Cha lacks evidence for any cold debris component.

Since the EF Cha disk spectrum does not resemble a featureless blackbody as in most debris disks (e.g., Chen et al. 2006), accurately determining dust temperatures is complicated by the presence of solid-state features. Moreover, accounting for these features may shift the best-fit dust temperatures and thus the inferred location of the dust. Nevertheless, we can conclude that EF Cha's disk emission is consistent with emerging from one or 
two debris belts at temperatures higher than the 0 torr water-ice condensation temperature $(\sim 170 \mathrm{~K} ; \sim 8.5$ AU from the star at LTE). Thus, EF Cha shows evidence for a warm debris disk with emission extending from the terrestrial zone to regions analogous to the solar system's asteroid belt, confirming earlier work by Rhee et al. (2007).

\subsection{Comparisons with Protoplanetary Disk Spectra and Typical Debris Disk Spectra: Empirical Limits on Dust Composition}

The mid-IR spectrum of the EF Cha debris disk shows evidence for many solid-state features. To investigate the composition of the dust disk, we first compare its emission to that from protoplanetary disks around Herbig AeBe stars and typical debris disks around 1-3 $M_{\odot}$ stars. Herbig AeBe star spectra exhibit a wide range of mid-IR spectral features, ranging from the comet-like spectrum of HD 100546 to the $10 \mu \mathrm{m}$ silicatefree spectrum of HD 169142 (Meeus et al. 2001; Grady et al. 2005).

The EF Cha spectrum is significantly different than that of the Herbig AeBe star HD 100546 (Figure 5) primarily in that it lacks strong polyaromatic hydrocarbon (PAH) emission features at $6.3 \mu \mathrm{m}, 7.7 \mu \mathrm{m}$, and $8.6 \mu \mathrm{m}$. Compared to other protoplanetary disk spectra studied by Meeus et al. (2001), EF Cha's disk shares the greatest similarity with disks like HD 150193 and HD 163296 labeled as "Group IIa," which have broad, "boxy" solid-state features at $\sim 10 \mu \mathrm{m}$ and generally lack clear PAH emission features at 6.2, 7.7, 8.6, and $11.2 \mu \mathrm{m}$. EF Cha shows that a secondary bump at $\sim 11.2 \mu \mathrm{m}$ - comparable in wavelength to a PAH emission feature such as that exhibited by HD 100546 but also easily explained by emission from olivine grains-lacks $\mathrm{PAH}$ emission features at shorter wavelengths.

Most debris disk spectra are featureless; for example, even when the dust is warm enough to emit significantly at $10 \mu \mathrm{m}$, debris disks usually lack the intrinsically strong $10 \mu \mathrm{m}$ silicate feature (e.g., Dahm \& Carpenter 2009; Morales et al. 2009). The lack of silicate features indicates that the dust grains are large with diameters $\geqslant 10 \mu \mathrm{m}$ (e.g., Chen et al. 2006). In sharp contrast, the EF Cha spectrum shows a complex $10 \mu \mathrm{m}$ peak and spectral structure longward of $\approx 15 \mu \mathrm{m}$, indicating that the disk contains many grains smaller than $10 \mu \mathrm{m}$. This behavior is qualitatively similar to that of other warm, highluminosity debris disks around young stars, such as those found around HD 113766A and HD 172555 (Lisse et al. 2008, 2009; Figure 5).

High-mass fractions of crystalline pyroxene can also be ruled out since they have multiple, narrow emission peaks in the $8-13 \mu \mathrm{m}$ range resulting in a spectrum far less smooth than observed. Likewise, the spectrum cannot contain as high of an abundance of crystalline olivine as that exhibited by comets and some Herbigs like the HD 100546 disk: such a high abundance would result in a narrow maximum at $\sim 9.7 \mu \mathrm{m}$ and a higher maximum at $\sim 11.2 \mu \mathrm{m}$ (see Lisse et al. 2007a), the opposite of what is observed. The general shape of the EF Cha spectrum is instead consistent with a high abundance of amorphous silicates and a secondary population of olivine. Amorphous silicates produce much broader $8-13 \mu \mathrm{m}$ peaks, resulting in a secondary maxima at $\sim 17-19 \mu \mathrm{m}$, and are abundant in Group IIa objects (e.g., Bouwman et al. 2001; Lisse et al. 2007a). However, superimposed upon this shape are peaks at 10, 11, 19, 23, 28, and $33 \mu \mathrm{m}$. The existence of these peaks indicates that additional species besides amorphous silicates are present.

\subsection{Comparisons with Mineralogical Models \\ 3.3.1. Description of a Mineralogical Model}

As shown by the empirical comparisons, the composition of mid-IR emitting dust from EF Cha's debris disk is dominated by amorphous silicates but must include high-mass fractions of other species. To identify these additional species, we fit the EF Cha IRS spectrum with a detailed mineralogical model based on laboratory thermal infrared emission spectra described in detail by Lisse et al. (2006, 2007a, 2007b, 2008). Below, we reiterate the key components of the model and describe its application to our data.

Thermal Dust Emission. The emission from distribution of dust is given by

$F_{\lambda, \text { model }}=\frac{1}{\Delta^{2}} \sum_{i, a} \int_{0}^{\infty} B_{\lambda}\left(T_{i}\left(a, r_{\star}\right)\right) Q_{\mathrm{abs}, \mathrm{i}}(a, \lambda) \pi a^{2} \frac{d n_{i}\left(r_{\star}\right)}{d a} d a$

where $T$ is the particle temperature for a particle of radius $a$ and composition $i$ at a distance $r$ from the central. The distance from Spitzer to the dust is $\Delta . B_{\lambda}$ is the blackbody radiance at wavelength $\lambda, Q_{\mathrm{abs}}$ is the emission efficiency of the particle of composition $i$ at wavelength $\lambda$, and $d n / d a$ is the differential particle size distribution (PSD) of the dust. The sum is over all species and all particle sizes. Our spectral analysis consists of calculating the flux for a model distribution of dust and comparing the result to the observed flux. The predicted flux from the model depends on the (1) dust composition (affects the location of spectral features), (2) particle sizes (affects feature-to-continuum contrast), and (3) the particle temperature (affects the strength of short versus long wavelength features).

Dust Composition. We use thermal laboratory emission spectra drawn from measurements for randomly oriented, $\mu$ m-sized powders, which yield $Q_{\mathrm{abs}}$ for each species. Sources for the emission spectra are detailed in the Supplemental Online Material from Lisse et al. (2006) and among others include contributions from the Jena spectral library (http://www.astro.uni-jena.de/Laboratory/OCDB) and from Koike (2000), Koike et al. (2002), and Chihara et al. (2002) for silicates. The library of material spectra was selected by their reported presence in interplanetary dust particles (IDPs), meteorites, in situ comet measurements, protoplanetary disks, and debris disks (see Lisse et al. 2006).

In total, the library includes spectra from 80 different species. The list of materials considered includes multiple silicates in the olivine and pyroxene class (forsterite, fayalite, clino- and orthoenstatite, augite, anorthite, bronzite, diopside, and ferrosilite). The model also includes phyllosilicates (such as saponite, serpentine, smectite, montmorillonite, and chlorite); sulfates (such as gypsum, ferrosulfate, and magnesium sulfate); oxides (including various aluminas, spinels, hibonite, magnetite, and hematite) $\mathrm{Mg} / \mathrm{Fe}$ sulfides (including pyrrhotite, troilite, pyrite, and niningerite); carbonate minerals (including calcite, aragonite, dolomite, magnesite, and siderite); water-ice, clean and with carbon dioxide, carbon monoxide, methane, and ammonia clathrates; carbon dioxide ice; graphitic and amorphous carbon; and neutral and ionized PAHs.

Dust Size Effects. We consider a dust size range of $0.1-1000 \mu \mathrm{m}$ in the model fits, with sharp emission features arising mainly from particles of $0.1-10 \mu \mathrm{m}$, and baseline continuum mainly from $10-1000 \mu \mathrm{m}$ particles. The particle size 


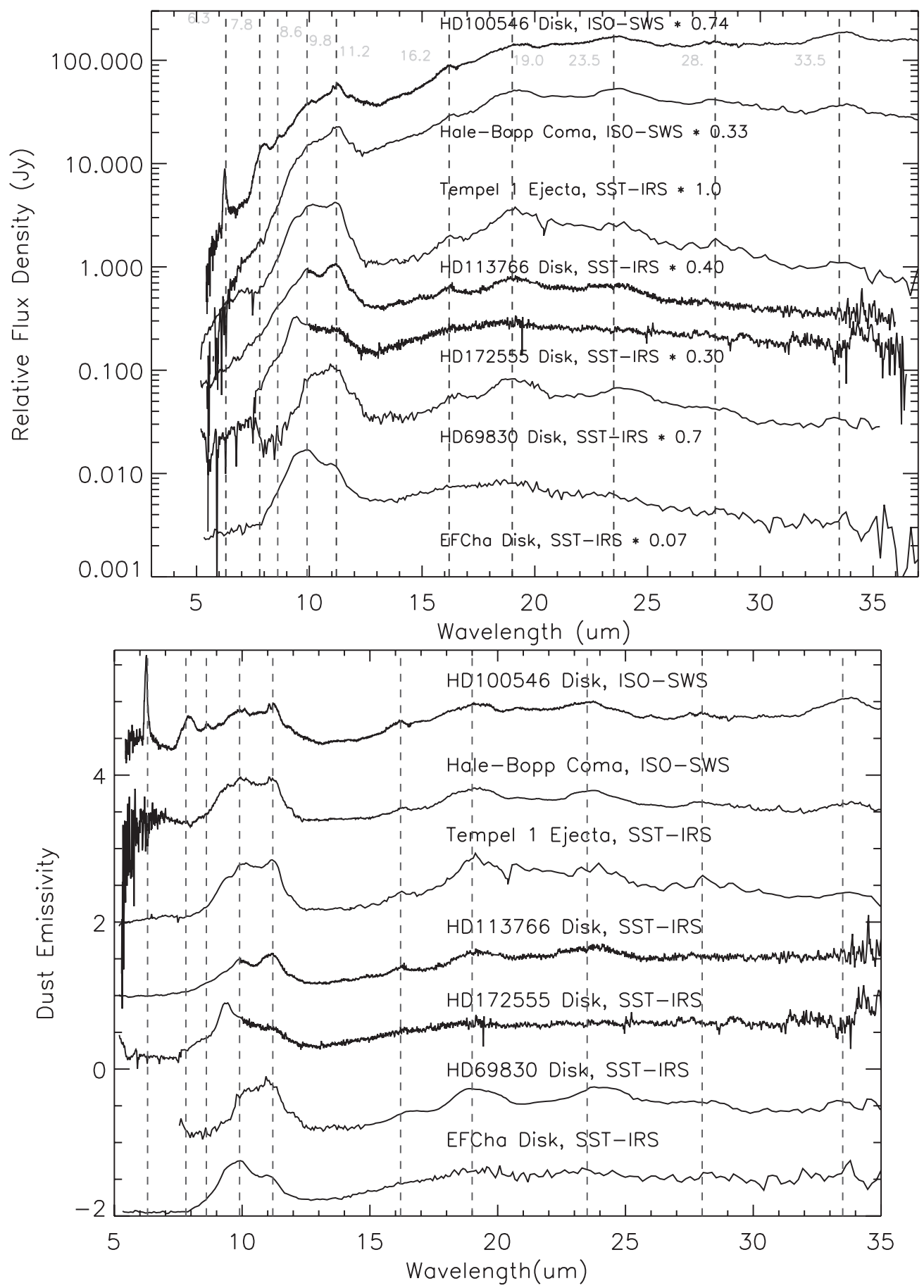

Figure 5. Top: spectra of a protoplanetary disk (HD 100546), Comet Hale-Bopp, the ejecta from Comet Tempel-1, and several high-luminosity debris disks-HD 113766, HD 172555, and HD 69830 compared to that for EF Cha (Lisse et al. 2008, 2009, 2007b). Wavelengths for PAH emission and other solid-state features are identified by vertical dashed lines. Bottom: spectra redisplayed in emissivity space to better contrast the feature to continuum flux ratios for each object.

affects the dust emissivity as

$$
1-\operatorname{Emissivity}(a, \lambda)=\left[1-\operatorname{Emissivity}(1 \mu \mathrm{m}, \lambda]^{a / 1 \mu \mathrm{m}}\right. \text {. }
$$

The PSD is fit at $\log$ steps in radius, i.e., at $0.1,0.2,0.5,1$, $2,5, \ldots, 10,20,50 \mu \mathrm{m}$, etc. Particles of the smallest sizes have emission spectra with very sharp features, and little continuum emission. Particles of larger sizes—greater than $\sim 10 \mu \mathrm{m}$ - tend to be optically thick and contribute only featureless continuum emission. Particles larger than $1000 \mu \mathrm{m}$ are not directly identifiable from our modeling and contribute no emission. We assume the same PSD for each species.
Dust Temperature. Dust particle temperatures are determined at the same log steps in grain radius used to determine the PSD. Dust temperature is a function of dust composition, size, and astrocentric distance. The highest temperature for the smallest particle size of each species is varied freely and is determined by the best fit to the data. The largest, optically thick particles $(1000 \mu \mathrm{m})$ are set to the LTE temperature, and the temperature of particles of intermediate sizes is interpolated between these two extremes by radiative energy balance.

Accuracy/Reliability of the Model and Fitting Method. This model has been successfully applied to both solar system 


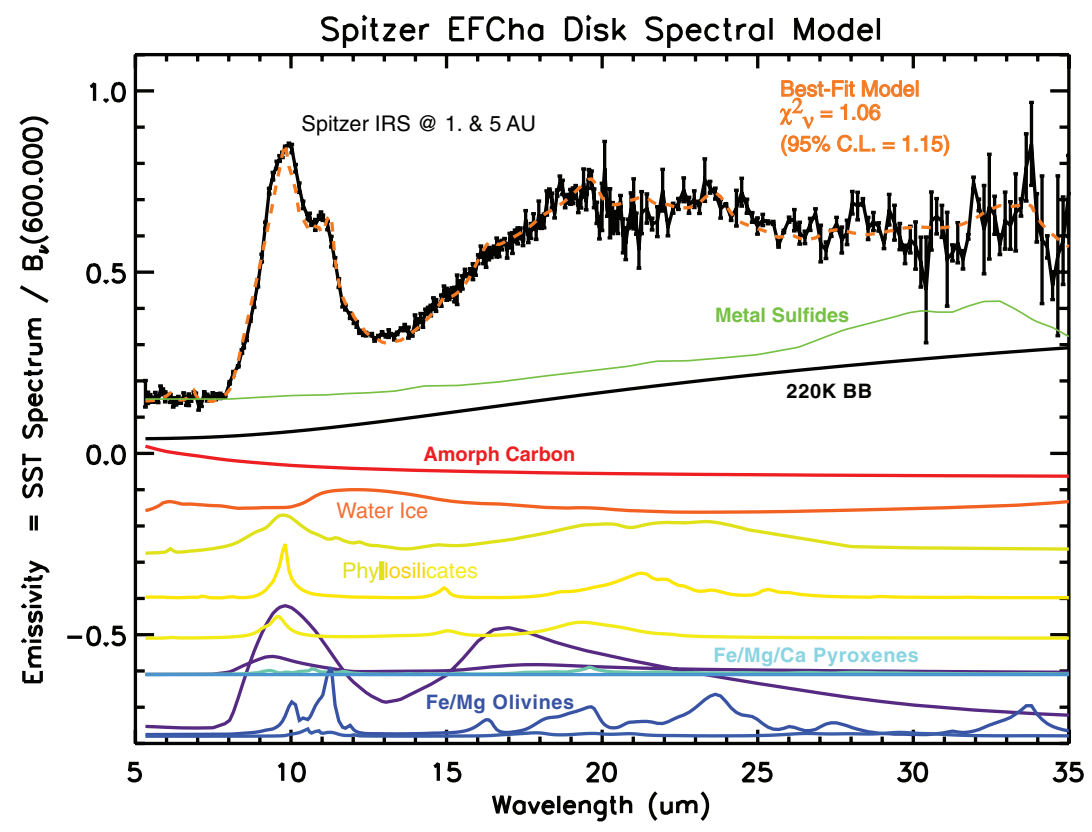

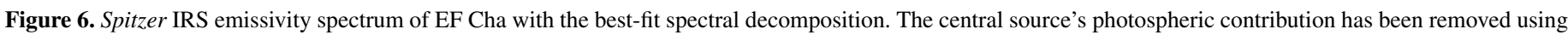

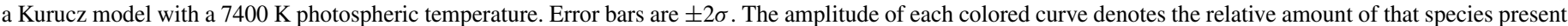

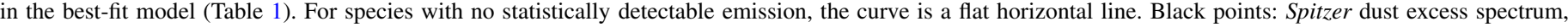

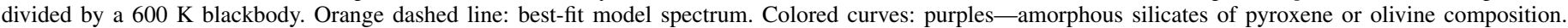

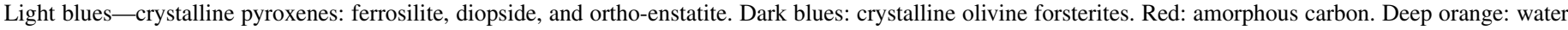

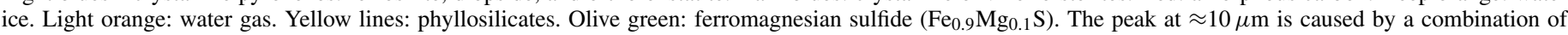

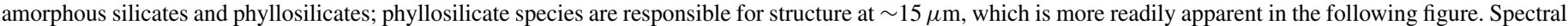
structure at $16-25 \mu \mathrm{m}$ is due mostly to forsterite.

(A color version of this figure is available in the online journal.)

objects and protoplanetary/debris disks around other stars. In particular, the model accounts for the emission spectra of comet 9P/Tempel 1 observed during the Deep Impact mission (A'Hearn et al. 2005; Lisse et al. 2006). Prior to the collision with the spacecraft, the comet's IR spectrum was near featureless, consistent with a coma dominated by large blackbody grains, similar to debris disk spectra around most stars. After collision, the spectrum showed more than 16 pronounced emission features, strong evidence for $\approx 10^{7} \mathrm{~kg}$ of small, submicron to micron-sized grains consisting of amorphous and crystalline silicates, amorphous carbon, carbonates, phyllosilicates, PAHs, water gas and ice, and sulfides (Lisse et al. 2006). As evidenced by the copious presence of fragile, volatile water-ice grains in the ejecta, the differences in the pre- and post-impact spectrum must be due to simple de-aggregation of weak, large $(\approx 100 \mu \mathrm{m}$ to $1 \mathrm{~cm}$ ), optically thick fractal dust particles in the delivered shock wave, rather than mineralogical transformation of the dust at high temperatures and pressures due to the delivered energy.

Direct compositional measurements from cometary sources also provide strong evidence that our modeling approach (1) is accurate for dust in the solar system and (2) can infer the composition of dust around other stars. A comparison of the derived Tempel 1 material composition with the materials found in the STARDUST sample return shows good agreement (Hanner \& Zolensky 2010). Moreover, the direct measurements of species within IDPs of cometary origin, in particular the GEMS (glass with embedded metal and sulfides) grains located within the matrices of "chondritic porous" IDPs, provide good evidence that cometary/meteoritic provides a good spectral match to "astronomical" silicates such as those inferred from spectra of protoplanetary and debris disks (Bradley et al. 1999).

For both Tempel 1 and debris disks around other stars like EF Cha, the dust responsible for mid-IR solid-state features is produced from collisions and must be freshly produced as it is small and easily removed from the system by radiation pressure, Poynting-Robertson drag, and stellar wind drag. Thus, applying our model to debris spectra from other stars can (1) identify the chemical composition of parent bodies from which the debris originates, (2) determine the sizes of debris particles, which may identify whether the debris must originate from largescale, catastrophic impacts, and (3) determine the range of dust temperatures. Lisse et al. (2008, 2009) argue that debris emission from disks surrounding HD 113766A and HD 172555 originates from debris created by large-scale collisional grinding/giant impacts associated with terrestrial planet formation and/or stochastic events such as that responsible for forming the Moon. Likewise, we here apply the model to the EF Cha spectrum to constrain the composition of parent bodies and investigate the origin of the debris.

Our method differs from other widely used methods in that it uses physically plausible emission measures from randomly oriented powders rather than theoretically derived Mie values. The free parameters of the model are the relative abundance of each detected mineral species, the temperature of the smallest dust particle of each species, and the value of the PSD. Best fits are found by a direct search through phase space-composition, temperature, and size distribution.

\subsubsection{Results of Fits From Our Mineralogical Model}

Figure 6 shows our fit to the EF Cha flux, presented in emissivity space (spectrum divided by an average Planck function of the entire dust population) for clarity, with the emissivity of individual constituent species labeled and shown as colored lines. Table 1 lists the relative abundances of each species. The best-fit model requires the presence of two warm dust belts, one with micron-sized dust at $600 \mathrm{~K}(\mathrm{LTE}$ at $\sim 430 \mathrm{~K}$, or $\sim 1.3 \mathrm{AU})$ 
Table 1

Composition of the Best-fit Model to the Combined Spitzer IRS EF Cha Spectrum

\begin{tabular}{|c|c|c|c|c|c|c|c|c|}
\hline Species & $\begin{array}{c}\text { Surface Area } \\
\text { (Weighted) }\end{array}$ & $\begin{array}{c}\text { Density } \\
\left(\mathrm{g} \mathrm{cm}^{-3}\right)\end{array}$ & M. W. & $\begin{array}{c}N_{\text {moles }} \\
\text { (rel.) }\end{array}$ & $\begin{array}{c}N_{\text {moles (Range) }} \\
\text { (rel.) }\end{array}$ & $\begin{array}{l}\text { Mass } \\
\text { (rel.) }\end{array}$ & $\begin{array}{l}\text { Model } T_{\text {Max }} \\
\text { (K) }\end{array}$ & $\begin{array}{c}\text { Model } \chi^{2} \\
\text { if not included }\end{array}$ \\
\hline \multicolumn{9}{|l|}{ Olivines } \\
\hline Amorph. Olivine $\left(\mathrm{MgFeSiO}_{4}\right)$ & 0.35 & 3.6 & 172 & 0.73 & {$[0.66,0.80]$} & 125.5 & 570 & 35.2 \\
\hline Forsterite $\left(\mathrm{Mg}_{2} \mathrm{SiO}_{4}\right)$ & 0.20 & 3.2 & 140 & 0.46 & {$[0.41,0.51]$} & 64.4 & 570 & 2.54 \\
\hline Fayalite $\left(\mathrm{Fe}_{2} \mathrm{SiO}_{4}\right)$ & 0.02 & 4.3 & 204 & 0.04 & {$[0.02,0.06]$} & 8.1 & 570 & 1.07 \\
\hline \multicolumn{9}{|l|}{ Pyroxenes } \\
\hline Amorph. Pyroxene $\left(\mathrm{MgFeSi}_{2} \mathrm{O}_{6}\right)$ & 0.06 & 3.5 & 232 & 0.09 & {$[0.08,0.10]$} & 20.9 & 570 & 1.54 \\
\hline Ferrosilite $\left(\mathrm{Fe}_{2} \mathrm{Si}_{2} \mathrm{O}_{6}\right)$ & 0.00 & 4.0 & 264 & 0.00 & {$[0,0]$} & 0 & 550 & 1.04 \\
\hline Diopside $\left(\mathrm{CaMgSi}_{2} \mathrm{O}_{6}\right)$ & 0.00 & 3.3 & 216 & 0.00 & {$[0,0]$} & 0 & 570 & 1.04 \\
\hline Ortho-enstatite $\left(\mathrm{Mg}_{2} \mathrm{Si}_{2} \mathrm{O}_{6}\right)$ & 0.02 & 3.2 & 200 & 0.03 & {$[0,0.04]$} & 6.0 & 570 & 1.07 \\
\hline \multicolumn{9}{|l|}{ Phyllosilicate Species } \\
\hline Smectite (Nontronite) & 0.10 & 2.3 & 496 & 0.05 & {$[0.04,0.06]$} & 24.8 & 570 & 3.10 \\
\hline Talc $\left(\mathrm{Mg}_{3} \mathrm{Si}_{4} \mathrm{O}_{10}(\mathrm{OH})_{2}\right)$ & 0.16 & 2.8 & 379 & 0.12 & {$[0.10,0.13]$} & 45.5 & 570 & 1.5 \\
\hline Saponite & 0.06 & 2.3 & 480 & 0.03 & {$[0.02,0.03]$} & 14.4 & 570 & 1.27 \\
\hline \multicolumn{9}{|l|}{ Carbonates } \\
\hline Magnesite $\left(\mathrm{MgCO}_{3}\right)$ & 0.02 & 3.1 & 84 & 0.07 & {$[0.06,0.08]$} & 5.88 & 570 & 1.09 \\
\hline Siderite $\left(\mathrm{FeCO}_{3}\right)$ & 0.00 & 3.9 & 116 & 0.00 & {$[0,0]$} & 0 & 570 & 1.04 \\
\hline \multicolumn{9}{|l|}{ Metal Sulfides } \\
\hline Niningerite $\left(\mathrm{Mg}_{10} \mathrm{Fe}_{90} \mathrm{~S}\right)$ & 0.27 & 4.5 & 84 & 1.45 & {$[1.31,1.60]$} & 121.8 & 570 & 7.82 \\
\hline \multicolumn{9}{|l|}{ Water } \\
\hline Water Ice & 0.08 & 1 & 18 & 0.44 & {$[0.40,0.49]$} & 7.92 & 190 & 4.66 \\
\hline Water Gas & 0.00 & 1 & 18 & 0.00 & {$[0,0]$} & 0 & 190 & 1.04 \\
\hline \multicolumn{9}{|l|}{ Organics } \\
\hline Amorph. Carbon & 0.09 & 2.5 & 12 & 1.88 & {$[1.69,2.07]$} & 22.6 & 715 & 6.73 \\
\hline $\mathrm{PAH}\left(\mathrm{C}_{10} \mathrm{H}_{14}\right)$ & 0.00 & 1.0 & 178 & 0.00 & {$[0,0]$} & 0 & $\mathrm{~N} / \mathrm{A}$ & 1.04 \\
\hline
\end{tabular}

Notes. The relative number of moles is calculated as follows: $N_{\text {moles }}(i) \sim \operatorname{Density}(i) / \operatorname{Molecular}$ Weight $(i) \times \operatorname{Surface}$ Area weighted $_{\text {The relative }}$ masses of each species are calculated from the product of $N_{\text {moles }}$ and the molecular weight. $N_{\text {moles }}$ (range) identifies the range of $N_{\text {moles }}$ from models that quantitatively provide a good fit to the data $\left(\chi^{2}<\chi^{2}(95 \%\right.$ C. L. $\left.)\right)$.

and one with large dust grains $(>20 \mu \mathrm{m})$ at an LTE temperature of $\sim 220 \mathrm{~K}(\sim 5 \mathrm{AU})$.

The best-fit model successfully reproduces the entire IRS spectrum and yields a small $\chi^{2}$ per degree of freedom $\left(\chi^{2} /\right.$ dof $\left.=1.06\right)$. To provide a statistical measure of the goodness of fit, we determine the $95 \%$ confidence limit given the number of degrees of freedom for a given reduced $\chi^{2}$ (see Lisse et al. 2008, 2009). The total number of free parametersincluding the number of relative abundances, the number of hottest particle temperatures, and the power law index-is 32 . Given the number of spectral points fit (272), the number of degrees of freedom is 240 . Based on these values, there is a $95 \%$ chance that a model with reduced $\chi^{2}$ less than 1.15 is a good predictor of the Spitzer data.

Given the excellent fit of the model from 5 to $38 \mu \mathrm{m}$, it is plausible that the chemical abundances included in the model comprise the dominant detectable species in the disk.

As suggested by the empirical comparisons, the EF Cha spectrum is olivine rich, containing large relative molar abundances of amorphous olivine and forsterite $\left(N_{\text {moles,rel }}=0.73\right.$ and 0.46 , respectively) responsible for the large width of the $10 \mu \mathrm{m}$ feature and the presence of a secondary peak at $\sim 11 \mu \mathrm{m}$. Fayalite also is present in low abundance. The model $\chi^{2}{ }_{v}$ is 1.07 with this species removed, lower than the $95 \%$ confidence limit, so evidence for this species is very weak. The emissivity slope and features at $\sim 20-35 \mu \mathrm{m}$ indicate the presence of water ice and metal sulfides, which are also abundant in the EF Cha disk ( $N_{\text {moles,rel }}=0.44$ and 1.45 , respectively). The spectrum contains a small abundance of pyroxene but lacks any clear evidence for water gas, carbonate, or PAH emission typical of primitive cometary material.
Interestingly, the EF Cha debris disk appears to contain a very high abundance of phyllosilicate species-smectite, talc, and saponite-responsible for the amplitude and shape of the pronounced peak at $10 \mu \mathrm{m}, 15 \mu \mathrm{m}$, and $20-23 \mu \mathrm{m}$ $\left(N_{\text {moles, rel }}=0.2\right.$ total $)$. Figure 7 , displaying disk emissivity with silicate contributions subtracted out, more clearly shows the importance of phyllosilicates and other major constituents like metal sulfides. In particular, phyllosilicates have singular sharp features and are required to reproduce the strong residual $10 \mu \mathrm{m}$ emission and complicated slope of the emissivity at $17-25 \mu \mathrm{m}$. $\mathrm{SiO}$ and silica have peaks at $8.8-9.2 \mu \mathrm{m}$ wavelengths, much shorter than $10 \mu \mathrm{m}$, and their inclusion in models clearly does not improve the $\chi^{2}$ parameter. Without the presence of phyllosilicate species, the $\chi^{2}{ }_{v}$ of the spectral fit is far worse- 3.1 for the removal of smectite alone. The range of abundances from any model passing the $95 \%$ confidence limit is narrow, typically $\sim 10 \%$ from the best-fit value.

Given the numerous strong solid-state features, the EF Cha disk must contain large numbers of small grains. The best-fit model includes a minimum grain size of $0.1 \mu \mathrm{m}$ : two orders of magnitude smaller than grain sizes for most IRS-studied debris disks. Moreover, the PSD for the best-fit model is $d n / d a \propto a^{-4.0}$. This steep distribution implies the presence of many more fine particles than for a collisional equilibrium size distribution $(d n /$ $\left.d a \propto a^{-3.5}\right)$.

While we can only directly sense the presence of grains with sizes $\lesssim 20 \mu \mathrm{m}$, grains up to millimeters in size have been found in debris disks (Maness et al. 2008). Moreover, micron-sized dust results from the collisional cascade induced from colliding, km-sized planetesimals and thus implies the presence of much larger bodies (e.g., Kenyon \& Bromley 2008; 


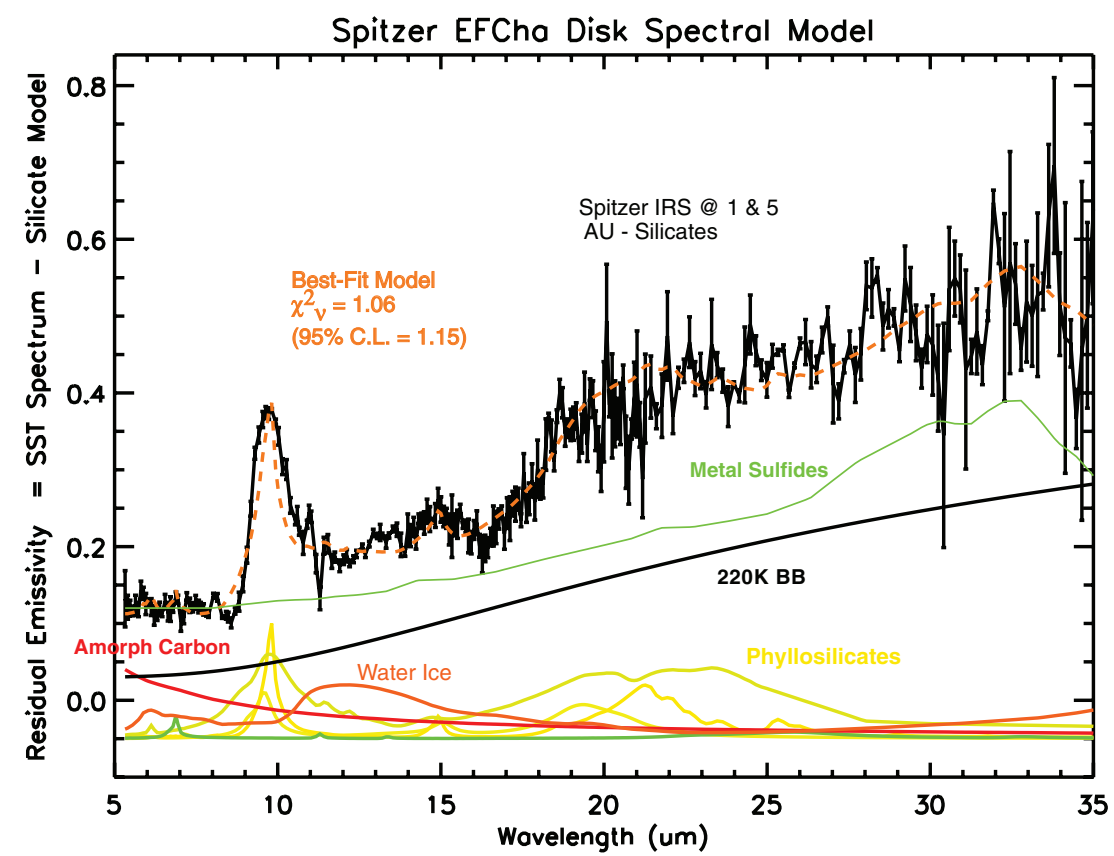

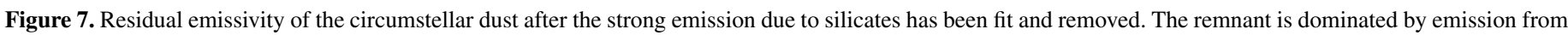

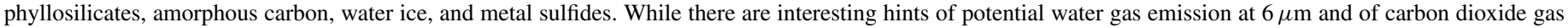
emission at $15 \mu \mathrm{m}$, neither of these features is statistically significant. Emission peaks at $9.8 \mu \mathrm{m}$ and $15 \mu \mathrm{m}$ due to phyllosilicate species are visible.

(A color version of this figure is available in the online journal.)

Wyatt et al. 2008; Krivov 2010). The size distribution of these fragments is predicted from theory to follow a power-law behavior roughly continuous with that for directly detectable debris dust (e.g., see Kenyon \& Bromley 2008). Therefore, we can get a rough estimate of the total mass of fragments involved in the collisions by extending the PSD up to larger sizes of order of $1 \mathrm{~mm}$.

Assuming a maximum grain size of $1000 \mu \mathrm{m}$, we estimate a total dust mass of $\sim 1.5 \times 10^{23} \mathrm{~g}$, comparable to the mass of an asteroid with a bulk density of $2.5 \mathrm{~g} \mathrm{~cm}^{-3}$ and a radius of $\sim 110 \mathrm{~km}$. This parent size is a lower limit since it is based on the total mass of grains with sizes less than $1 \mathrm{~mm}$. Larger, $100-1000 \mathrm{~km}$-sized bodies are likely present since they are required to initiate and sustain the collisional cascade that produces the $<1 \mathrm{~mm}$-sized debris (e.g., Kenyon \& Bromley 2008, 2010).

The large number of small grains must require a replenishment source since they would otherwise be rapidly removed by radiation pressure (e.g., Backman \& Paresce 1993; Chen et al. 2006). Assuming a stellar mass of $1.9 M_{\odot}$, a stellar luminosity of $9.7 L_{\odot}$, and a grain density of $3 \mathrm{~g} \mathrm{~cm}^{-3}$, the blowout size limit for spherical grains in EF Cha, using the equation in Currie \& Kenyon (2009), is $\sim 2 \mu \mathrm{m}$. Thus, the EF Cha debris disk contains a high mass of dust dominated by particles comparable to or smaller than those dominating typical debris disks and also far smaller than the nominal blowout size limit for the system. Along with the stability of the 1983-2007 IRAS/MSX/ Spitzer photometry, the copious amount of small dust indicates that there must be a source for small particle replenishment.

\subsubsection{Tests of Phyllosilicate Identification}

Additional tests are required to more reliably constrain the presence of individual species responsible for the EF Cha debris emission, especially phyllosilicate species. First, although our best-fit model includes phyllosilicate species, our solution is not unique if a phyllosilicate-free model reproduces the observed spectrum within the confidence limit we adopt to identify goodfitting models (the $95 \%$ confidence limit). Second, the signatures from phyllosilicate species and other species like metal sulfides are weaker than the amorphous silicates also present in the disk. Since they are weaker their presence is more likely to be falsely identified in real spectra with random noise patterns.

To address these issues, we carried out two additional sets of model fits. First, we reran the fits with all phyllosilicate species removed, in order to determine whether all good-fitting models require their presence. Second, we fit our models to two independent EF Cha spectra obtained concurrently at separate IRS nod positions. Comparing the two model results constrains the sensitivity of our identification of different species to photon noise. Moreover, since the spectra at different nod positions are extracted from different regions on the IRS detector, fits to separate nod positions test the sensitivity of our identifications to different IRS systematic noise contributions.

Figure 8 displays the best-fit model obtained with contributions from all phyllosilicate species removed. If the best-fit model fares only slightly worse in reproducing the inferred emissivity with and without silicate contributions, then the EF Cha disk composition is underdetermined and we cannot constrain the existence and mass fractions of phyllosilicate species. As shown by Figure 8 , the $\chi^{2}$ of the best-fit model lacking phyllosilicates is substantially worse than for the model including phyllosilicates. While the general shape of the emissivity is accurately reproduced, the phyllosilicate-free model predicts a strong peak at $\sim 11.25 \mu \mathrm{m}$, which is not observed, and incorrectly predicts the flux ratios of peaks at $10 \mu \mathrm{m}$ and $11 \mu \mathrm{m}$. The model also significantly underpredicts the flux at $23 \mu \mathrm{m}$.

The silicate-subtracted emissivity even more strikingly reveals the failure of our phyllosilicate-free model (Figure 9). The model emissivity is relatively flat from $8 \mu \mathrm{m}$ to $11 \mu \mathrm{m}$. With the 


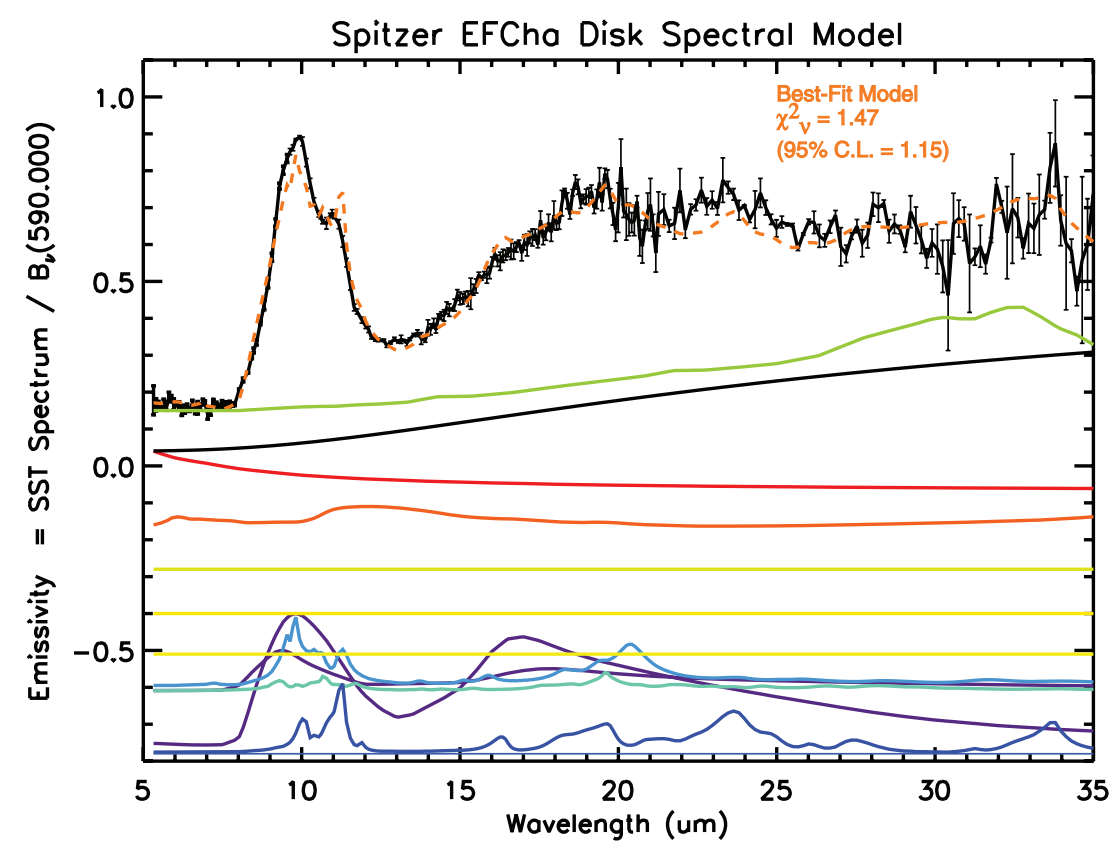

Figure 8. Same as Figure 6 except with phyllosilicates removed from the model mix of materials and the Spitzer data refitted. (A color version of this figure is available in the online journal.)

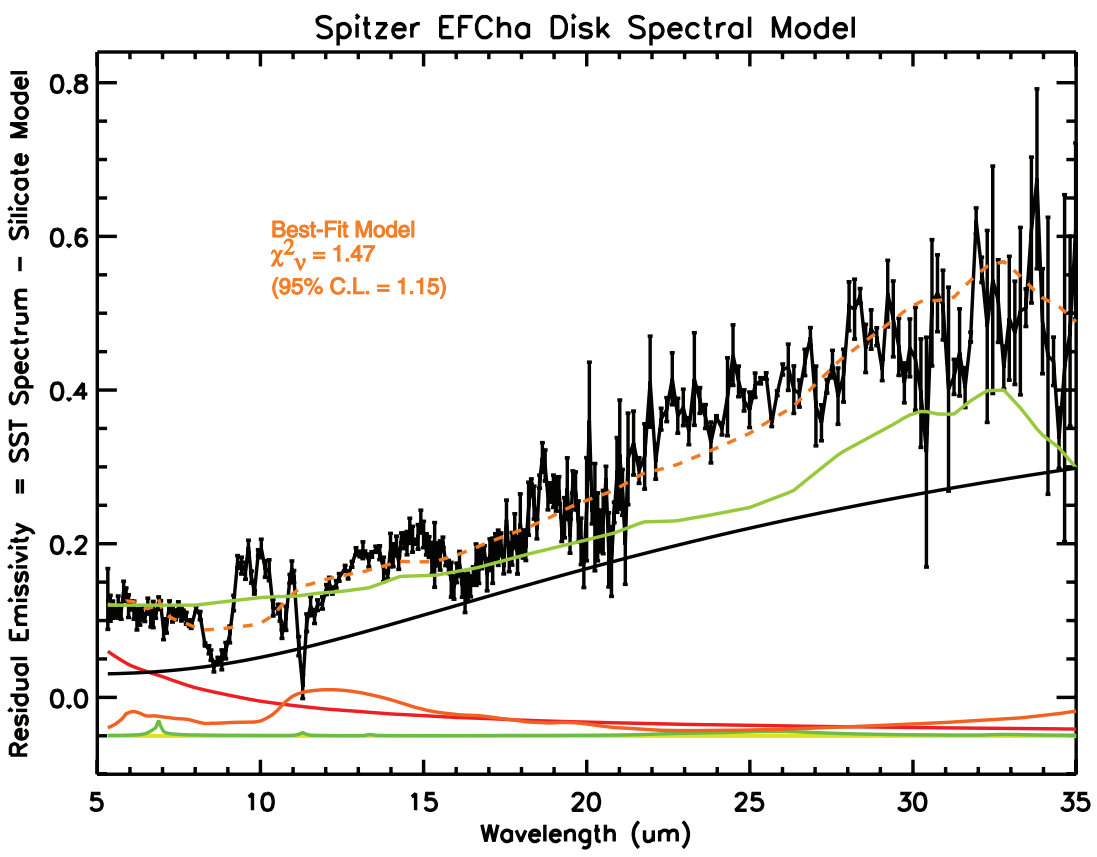

Figure 9. Same as Figure 7 except with phyllosilicates removed from the model mix of materials and the Spitzer data refitted.

(A color version of this figure is available in the online journal.)

silicate contribution from the model subtracted out, the observed emissivity has local maxima at $\sim 9.5-10 \mu \mathrm{m}$ and $23 \mu \mathrm{m}$ well above that of the model. Local minima at 8-9 $\mu \mathrm{m}$ and 11-12 $\mu \mathrm{m}$ lie well below that of the model. The model also lacks the structure apparent at $\sim 14-20 \mu \mathrm{m}$ in the silicate-subtracted emissivity from the data. Compared to the phyllosilicate-free model, the best-fit model including phyllosilicates clearly reproduces the emissivity far better. Quantitatively, the phyllosilicate-free model can be ruled out as a good-fitting model. It has a reduced $\chi^{2}$ value $\sim 40 \%$ larger $\left(\chi^{2} /\right.$ dof $=1.47$ for 240 degrees of freedom). This difference in $\chi^{2}$ is statistically significant, since the probability that the phyllosilicate-free model fits the Spitzer data is $\sim 0.0002$.

Table 2 summarizes the modeling results for our fits to the separate nod positions. The number of moles and weighted surface area for the species as a whole show exceptional agreement with the results derived for the combined spectrum. While a few of the abundances do vary-e.g., for amorphous olivine, amorphous pyroxene, smectite-they do so by less than the $10 \%-20 \%$ systematic uncertainties on the abundances in all cases. While only one nod position requires the presence of a small amount of ortho-enstatite, the presence/absence of other 
Table 2

Composition of the Best-fit Model to the Spitzer IRS EF Cha Spectrum for Different Nod Positions

\begin{tabular}{|c|c|c|c|c|c|}
\hline Species & $\begin{array}{c}\text { Surface Area } \\
\text { (Weighted) }\end{array}$ & $\begin{array}{c}\text { Density } \\
\left(\mathrm{g} \mathrm{cm}^{-3}\right)\end{array}$ & M. W. & $\begin{array}{c}N_{\text {moles }} \\
\text { (rel.) }\end{array}$ & $\begin{array}{c}\text { Model } T_{\text {Max }} \\
(\mathrm{K})\end{array}$ \\
\hline \multicolumn{6}{|l|}{ Olivines } \\
\hline Amorph. Olivine $\left(\mathrm{MgFeSiO}_{4}\right)$ & $0.37 / 0.35$ & 3.6 & 172 & $0.77 / 0.73$ & 570 \\
\hline Forsterite $\left(\mathrm{Mg}_{2} \mathrm{SiO}_{4}\right)$ & $0.20 / 0.20$ & 3.2 & 140 & $0.46 / 0.46$ & 570 \\
\hline Fayalite $\left(\mathrm{Fe}_{2} \mathrm{SiO}_{4}\right)$ & $0.01 / 0.03$ & 4.3 & 204 & $0.02 / 0.06$ & 570 \\
\hline \multicolumn{6}{|l|}{ Pyroxenes } \\
\hline Amorph. Pyroxene $\left(\mathrm{MgFeSi}_{2} \mathrm{O}_{6}\right)$ & $0.06 / 0.05$ & 3.5 & 232 & $0.09 / 0.08$ & 570 \\
\hline FerroSilite $\left(\mathrm{Fe}_{2} \mathrm{Si}_{2} \mathrm{O}_{6}\right)$ & $0.00 / 0.00$ & 4.0 & 264 & $0.00 / 0.00$ & 550 \\
\hline Diopside $\left(\mathrm{CaMgSi}_{2} \mathrm{O}_{6}\right)$ & $0.00 / 0.00$ & 3.3 & 216 & $0.00 / 0.00$ & 570 \\
\hline Ortho-enstatite $\left(\mathrm{Mg}_{2} \mathrm{Si}_{2} \mathrm{O}_{6}\right)$ & $0.00 / 0.02$ & 3.2 & 200 & $0.00 / 0.03$ & 570 \\
\hline \multicolumn{6}{|l|}{ Phyllosilicate Species } \\
\hline Smectite (Nontronite) & $0.09 / 0.11$ & 2.3 & 496 & $0.04 / 0.05$ & 570 \\
\hline Talc $\left(\mathrm{Mg}_{3} \mathrm{Si}_{4} \mathrm{O}_{10}(\mathrm{OH})_{2}\right)$ & $0.16 / 0.16$ & 2.8 & 379 & $0.12 / 0.12$ & 570 \\
\hline Saponite & $0.07 / 0.07$ & 2.3 & 480 & $0.03 / 0.03$ & 570 \\
\hline \multicolumn{6}{|l|}{ Carbonates } \\
\hline Magnesite $\left(\mathrm{MgCO}_{3}\right)$ & $0.01 / 0.01$ & 3.1 & 84 & $0.07 / 0.07$ & 570 \\
\hline Siderite $\left(\mathrm{FeCO}_{3}\right)$ & $0.00 / 0.00$ & 3.9 & 116 & $0.00 / 0.00$ & 570 \\
\hline \multicolumn{6}{|l|}{ Metal Sulfides } \\
\hline Niningerite $\left(\mathrm{Mg}_{10} \mathrm{Fe}_{90} \mathrm{~S}\right)$ & $0.28 / 0.27$ & 4.5 & 84 & $1.5 / 1.45$ & 570 \\
\hline \multicolumn{6}{|l|}{ Water } \\
\hline Water Ice & $0.08 / 0.08$ & 1 & 18 & $0.44 / 0.44$ & 190 \\
\hline Water Gas & $0.00 / 0.00$ & 1 & 18 & $0.00 / 0.00$ & 190 \\
\hline \multicolumn{6}{|l|}{ Organics } \\
\hline Amorph. Carbon & $0.09 / 0.09$ & 2.5 & 12 & $1.88 / 1.88$ & 715 \\
\hline PAH $\left(\mathrm{C}_{10} \mathrm{H}_{14}\right)$ & $0.00 / 0.00$ & 1.0 & 178 & $0.00 / 0.00$ & $\mathrm{~N} / \mathrm{A}$ \\
\hline
\end{tabular}

Notes. The two entries for the weighted surface area and the number of moles for the first nod position and then the second nod position. With the exception of ortho-enstatite, our identification of species from modeling the combined spectrum is not affected by the noise characteristics of the spectra.

species is completely consistent from one nod position to the other.

Most importantly, fits to both nod positions require the presence of the three phyllosilicate species identified from fitting the combined spectrum. The total weighted surface area for these species varies little between nod positions $(0.32$ versus $0.34)$, and as a result the molar abundances vary little $(0.19$ versus 0.20 ). Thus, our quantitative estimate of the phyllosilicate contribution to the EF Cha debris disk does not depend on the nod position used. Since fitting to different nod positions probes the sensitivity of our results to noise characteristics, our results show that our identification and abundance estimate of phyllosilicate species is robust against the influence of random and systematic noise.

At a minimum, we can conclude that some suite of species whose emissivities are extremely similar to the laboratorymeasured emissivities for our suite of finely powdered phyllosilicate species are abundantly present in the EF Cha debris disk. Given the complexity of the phyllosilicate family and the large range of variants for a given phyllosilicate (e.g., the interchangiability of $\mathrm{Ca}$ and $\mathrm{Al}$, as well as $\mathrm{Fe}$ and $\mathrm{Mg}$ ), it is not totally surprising that we may not yet have the exact laboratory match for the EF Cha spectral signature. Moreover, the precise shape of the spectral features depends on the exact details of how the minerals in the parent bodies are fragmented, combined, heated, and cooled. A priori, we cannot rule out the existence of species not included in our chemical model that can mimic the emissivity of phyllosilicate species. These uncertainties preclude any proof that the EF Cha disk must contain phyllosilicate material.

However, the presence of abundant phyllosilicate material in a variety of solar system contexts-e.g., carbonaceous chondritic meteorites, Comet 9P/Tempel-1, main belt asteroids (Tomeoka \& Ohnishi 2010; Morlok et al. 2010; Lisse et al. 2006; Vilas \& Gaffey 1989; Bus \& Binzel 2002)—lends powerful support to our inference that the species in question is indeed phyllosilicate species. If the species in question is not phyllosilicates, chemical processes must have been able to remove these species selectively and thoroughly, which seems implausible given the wealth of other species present. Given the extensive library of species used for our spectral fitting and rejected as poorly matching (Lisse et al. 2006), it also seems unlikely that some other species is mimicking the phyllosilicate species' signatures.

\subsection{Comparisons to the Mineralogy, Dust Sizes, and Atomic Abundances of Comet Tempel-1 Ejecta and Warm, High-Luminosity Debris Disks}

To provide an additional framework for interpreting our results, here we compare the EF Cha dust mineralogy, dust sizes, and atomic abundances to those for the Comet Tempel1 ejecta and warm, young debris disks we have modeled in previous papers: HD 113766A and HD 172555 (Lisse et al. 2006, 2007a, 2008, 2009) all of which demonstrate strong mid-IR features like EF Cha. For both Comet Tempel-1 and HD 113766A, the 9-12 $\mu \mathrm{m}$ flux exhibits a broad plateau with two peaks of equal amplitude at $\sim 10 \mu \mathrm{m}$ and $11 \mu \mathrm{m}$ due to abundant forsterite, enstatite, ferrosilite, and (to a lesser extent) amorphous olivine and pyroxene. Best-fit models for the Comet Tempel-1 ejecta and HD 113766A also require the phyllosilicate species smectite nontronite. While HD 172555 has a single sharp peak, it is at $\sim 9 \mu \mathrm{m}$ with a gradual decline in flux from 10 to $12 \mu \mathrm{m}$ (Lisse et al. 2009). Our modeling identifies this 
peak as due to a combination of amorphous silica, tektite, and $\mathrm{SiO}$ gas. EF Cha's spectrum is different from all of these, exhibiting a strong $10 \mu \mathrm{m}$ phyllosilicate peak superimposed upon a smoother $10 \mu \mathrm{m}$ amorphous silicate feature and the $9.3,9.8$, and $11.2 \mu \mathrm{m}$ sharp features of crystalline olivine \& pyroxene. The phyllosilicate molar abundance for nontronite, talc, and saponite combined is far higher than that for HD 113766 A or Comet Tempel-1 $\left(N_{\text {moles,rel }}=0.20\right.$ versus 0.03 and 0.07 ) and thus much more significant.

The abundant small grain population for EF Cha is most consistent with a large mass fraction of debris emission being actively produced, either by a single, massive catastrophic collision followed by grinding of the fragments or an abnormally high, steady collision rate. The agreement in flux density between the 1983 IRAS, 1996 MSX, and 2007 Spitzer data is at least consistent with the emission being maintained at a high level for 20 years or more. Timescales for grain removal by Poynting-Robertson drag are longer than this limit (e.g., Backman \& Paresce 1993; Chen et al. 2006; Currie \& Kenyon 2009); however, removal timescales for the smallest grains due to radiation pressure are more comparable (e.g., orbital timescales of 1-100 yr), indicating that at least the smallest grains may require active replenishment. Similarly, the presence of small grains and very steep PSD is qualitatively consistent with that seen in the Tempel 1 ejecta produced by the Deep Impact collision and with fine dust created by a hypervelocity impact found around HD 172555.

Like HD 113766A and Comet Tempel-1, the EF Cha spectrum may indicate the presence of water ice. HD 113766A has two debris belts beyond the ice line and a warm one inside of it. Our spectral modeling suggests that EF Cha contains a warm belt well interior to the ice line and second one close to the ice line, at $220 \mathrm{~K}$ at 7.6 AU. Water ice present in the interior EF Cha belt, in a radiation field with a local equilibrium temperature of $\sim 600 \mathrm{~K}$, should be very quickly vaporized and thus not present in the mid-IR spectrum unless it has been freshly ejected from a parent body. Collisions producing the observed water ice then either must have occurred very recently or must be occurring in the outer, $220 \mathrm{~K}$ belt. The belt at $7.6 \mathrm{AU}$ may even provide a reservoir for some icy dust concentrated in the warm, terrestrial zone belt. In either case, the presence of water ice requires an active replenishment source. Moreover, if water ice is being ejected from parent bodies in the $\sim 7.6$ AU region near the ice line, the EF Cha spectrum may show evidence for faint, water gas emission. If water ice is indeed present there must be an active replenishment and a continual release of gaseous water. We do not detect water gas at a statistically significant level (see Section 3.1), although the expected amount is difficult to quantify. Thus, we do not rule out its presence at the observed signal-to-noise level.

Within the context established by data for comets, meteorites, protoplanetary disks, and warm debris disks, EF Cha's olivine mass fraction indicates that the system bridges a gap between relatively primitive unprocessed solids and heavily processed bodies (Figure 10, top panel). Primitive bodies coming in contact with water, and thus aqueously altered, gain in their mass fractions of olivine and olivine-to-pyroxene ratios. ${ }^{5}$ Differentiated bodies and carbon-rich bodies processed at high temperatures have an even higher abundance of olivine and lie in the upper right-hand corner of the plot. Samples with a low percentage

\footnotetext{
5 Here, we include phyllosilicates in the pyroxene mass fraction, assuming these species are formed by alteration of pyroxene by warm, reactive water (see Tomeoka 1990; Morlok et al. 2010; Nagahara \& Ozawa 2011).
}

and relative fraction of olivine occupy the lower left corner and include comets (SW3, Holmes, Wild 2, and Tempel 1) and protoplanetary disks (HD 100546, HD 98800, HD 163296; Sitko et al. 2008; Lisse et al. 2006, 2007a, 2008). Those in the upper right are consistent with differentiated and/or pyrolyzed bodies include ancient debris disks surrounding white dwarfs (GD362, G29-38) and the centaur SW-1 (Stansberry et al. 2004; Reach et al. 2009b; Jura 2006; Jura et al. 2009). In this regard, EF Cha is roughly halfway between Hale-Bopp/Tempel-1 and HD 113766, which respectively identify the boundaries of the primitive, unprocessed group and the highly processed, differentiated/pyrolyzed group.

The bottom panel of Figure 10 compares atomic abundances for the EF Cha debris disk to that for the Sun and for other debris disks, protoplanetary disks, and comets (e.g., see Lisse et al. 2008). Like other debris/protoplanetary disks and comets-HD 172555, $\eta$ Corv, HD 100546, etc.- - the EF Cha emitting debris has heavily subsolar abundances of hydrogen, carbon, and oxygen. EF Cha has solar abundances of the main refractory elements $\mathrm{Si}, \mathrm{Mg}, \mathrm{Fe}$, and $\mathrm{Al}$, a subsolar abundance of calcium, and supersolar abundances of sulphur. Overall, EF Cha's atomic abundance pattern shows the strongest similarity with that of HD 113766A, another luminous warm debris disk of comparable age whose spectrum shows evidence for massive terrestrial-zone collisions. To first order, EF Cha's debris dust may be thought of as a more unprocessed/amorphous, water-rich analog to HD 113766 A's.

\subsection{Interpretation of Modeling Results}

Phyllosilicate species are tracers for the past presence of warm reactive water, since they are produced by the aqueous alteration of anhydrous rock (e.g., Tomeoka 1990). Based on our modeling results, the emitting dust from EF Cha's debris disk plausibly includes a high abundance of phyllosilicate grains and smaller abundances of water ice and metal sulfides in the terrestrial zone released from parent bodies by collisions. Because phyllosilicates should slowly decompose at temperatures of $T \sim$ 600-700 K (Akai 1992; Nagahara \& Ozawa 2011), they can exist within much of the terrestrial zone on the order of years after release from their parent body. However, stable low-pressure phyllosilicate formation over millions of years, whether from solar nebula condensation or from aqueous alteration in the interior of an asteroidal water-rich body, requires bodies that were originally formed/condensed at temperatures $<300-350$ K (e.g., Fegley \& Prinn 1989; Wasson 2008; Nagahara \& Ozawa 2011). Just as the presence of icy parent bodies scattered into the terrestrial zone from beyond the ice line that can be inferred from water-ice spectral features, we can conclude that the current warm dust belt arises in part from solids imported from cold $(<300 \mathrm{~K})$ regions, more distant from the star. The composition of the parent bodies responsible for EF Cha's debris emission is then likely not characteristic of planetesimals that, $\sim 5-10 \mathrm{Myr}$ prior, grew from micron to millimeter-sized grains in the warm, terrestrial regions of its protoplanetary disk. Rather, the current debris disk composition arises in part from solids imported from colder regions, more distant from the primary star.

The large abundance of small grains, evidence for high collision rates, and atomic abundance pattern makes EF Cha's debris disk comparable to the high-luminosity, warm debris disks around HD 113766A. However, its chemical composition is very different, given its high phyllosilicate abundance and moderately low pyroxene abundance consistent with aqueously altered primitive material. If large-scale terrestrial planet 


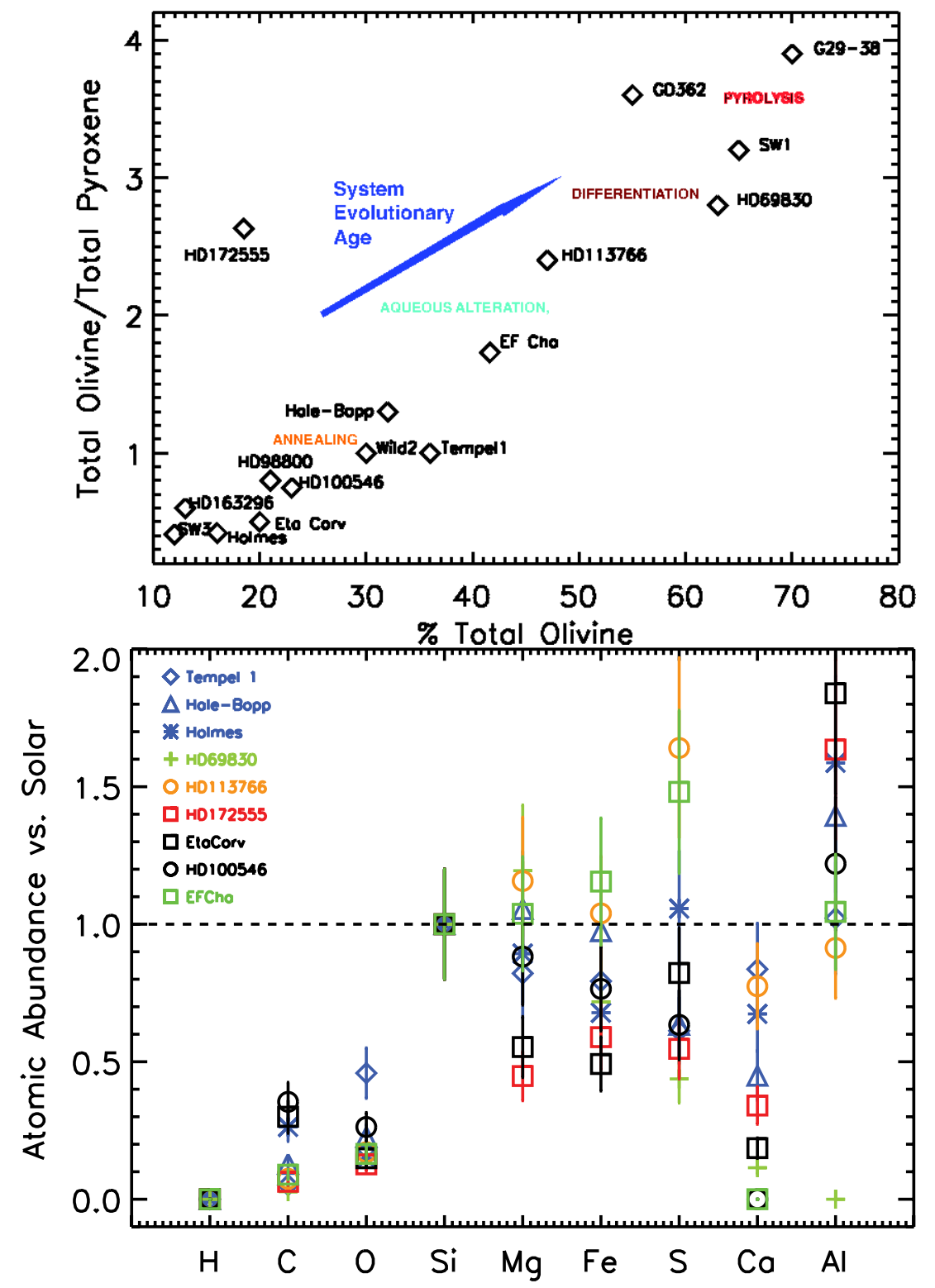

Figure 10. Top: olivine vs. pyroxene mass fraction for EF Cha compared to that for other debris disks, protoplanetary disks, comets, and asteroids. The distribution of ratios defines a system evolutionary sequence from unprocessed, primitive rock to highly processed, aqueously altered, and pyrolyzed rock. Bottom: atomic abundances relative to solar for EF Cha and other debris disks, protoplanetary disks, and comets.

(A color version of this figure is available in the online journal.)

formation is occurring in EF Cha like HD 113766A, then it is occurring from a feedstock of water-rich C- or D-type asteroids, not water-poor S-type asteroids as inferred by Lisse et al. (2008) for HD113766A. Combining our disk chemistry and grain size results, we conclude that the EF Cha debris disk traces (1) a high level of terrestrial-zone collisions between bodies which, at least in part, (2) originated from beyond the terrestrial zone, and (3) have processed phyllosilicates from primordial silicates at some time in the past.

\section{DISCUSSION}

\subsection{The Importance of EF Cha's Phyllosilicate Species}

The detection of abundant phyllosilicates in EF Cha's debris disk is of particular interest to studies of the early solar system and the habitability of terrestrial planets. Phyllosilicate formation results from the reaction between anhydrous rock and water and thus its presence in a debris disk requires an abundant source of water-rich planetesimals. Meteoritic data show evidence for numerous "fine-grained" phyllosilicate rims surrounding chondrules and calcium-aluminum-rich inclusions in carbonaceous chondrites and meteorites-i.e., the parent bodies that are a likely source for Earth's water (e.g., Robert et al. 2000) — with abundances similar to phyllosilicate-rich asteroids in the outer asteroid belt $(r>2.5 \mathrm{AU}$; Gradie \& Tedesco 1982; Bell et al. 1989). If phyllosilicate species are detected in debris disk spectra, their presence then requires an abundant source of water-rich planetesimals. Thus, the presence of phyllosilicates in the terrestrial zone of disks surrounding young stars may trace water delivery in young planetary systems.

Moreover, because phyllosilicates trace the existence of water in disks, they may be important signposts for the future development of primitive life. Indeed, the presence of abundant phyllosilicates in the most ancient regions of Mars' surface has 
been used in part to argue that Mars may have once supported habitable conditions (Bibring et al. 2006). Surface regions rich in phyllosilicates may be prime locations for finding early, primitive organisms (Farmer \& Des Marais 1999; Orofino et al. 2010).

\subsection{Phyllosilicate Production and Water Delivery}

Planetesimal collisions are responsible for releasing small grains that allow us to identify phyllosilicate species. However, it is important to emphasize that the collisions between anhydrous rock and hydrous planetesimals are not likely for the production of the phyllosilicate species. Impact processing tends to devolatilize planetesimals and should convert any phyllosilicates on the surface back to pyroxene (e.g., Morlok et al. 2010; see Section 4.3 of this work). Instead, phyllosilicates species are formed from the combination of pyroxene and warm, reactive water, where the water likely resulted from ice melted internal heating due to ${ }^{26} \mathrm{Al}$ decay (e.g., Desch \& Leshin 2004; Merk \& Prialnik 2006; and references therein). Thus, it is more likely that the collisions are simply revealing aqueously altered rock as opposed to being a process that actively alters anhydrous rock on the planetesimals' surfaces.

Recent studies of the solar system's main belt asteroids have yielded precisely this - detections of water and aqueously altered products (e.g., Campins et al. 2010; Rivkin \& Emery 2010). The total dust mass required to reproduce the EF Cha disk emission is comparable in mass to a $110 \mathrm{~km}$ radius asteroid: a parent body of comparable size and density is then massive enough to have contained liquid water (Merk \& Prialnik 2006). If our conclusion about the presence of phyllosilicate species is correct, it implies that asteroidal processing such as that identified from solar system asteroids may be quite advanced by 10 Myr.

The mechanism for the delivery of water-rich planetesimals to the terrestrial zone (needed for phyllosilicate production) is less unclear, although there are several plausible mechanisms. After the planetesimal population in the outer disk has been significantly depleted, the remaining planetesimals could be dynamically scattered inward and incorporated into forming terrestrial planets, especially after the solar nebula has dissipated (Goldreich et al. 2004; Ford \& Chiang 2007; Matsumura et al. 2010). Water-rich planetesimals could also be rapidly delivered from asteroid belt regions to the terrestrial zone by gas drag during the protoplanetary disk phase and later incorporated into larger bodies (e.g., Ciesla \& Lauretta 2005), or form nearly in situ if the disk is cold. In other systems, volatile-rich planets formed beyond the ice line could fail to become the cores of gas giants and migrate into the terrestrial zone through planet-disk or planet-planet interactions (Kuchner 2003).

From the standpoint of our mineralogical analysis, all of these scenarios are equivalent. EF Cha's disk is a debris disk, not a protoplanetary disk. Therefore, we do not know exactly when or how the water needed for phyllosilicate production was first introduced to the terrestrial zone in EF Cha.

\subsection{The Detectability of Phyllosilicate Species in Protoplanetary Disks and Other Debris Disks}

A more answerable question is why so far only EF Cha, among all debris disks and protoplanetary disks, shows strong evidence for abundant phyllosilicates. First, detecting phyllosilicate species with Spitzer requires dust warm enough to effectively emit at the $10-25 \mu \mathrm{m}$ with sufficiently small sizes
$(<10 \mu \mathrm{m})$ to show solid-state features. Both models for terrestrial planet formation and Spitzer surveys indicate that warm debris dust around young stars is rare because the processes that can make the dust are fleeting and/or yield too few small collisional fragments for detectable solid-state features (e.g., Kenyon \& Bromley 2004; Chen et al. 2006; Currie et al. 2007a; Gorlova et al. 2007). Thus, the subset of young debris disks that could possibly yield detectable mid-IR features of phyllosilicates is small compared to the sample of all young debris disks.

Second, experiments on phyllosilicate-rich chondritic meteorites indicate that collisions cannot be too high energy, else the phyllosilicates will be destroyed. Morlok et al. (2010) subjected matrix sections of the phyllosilicate-rich Murchison CM chondritic meteorite to projectile collisions with peak pressures ranging between 10 and $49 \mathrm{GPa}$. The weakly shocked samples ( $\sim 10 \mathrm{GPa})$ corresponding to low-energy collisions have spectra dominated by phyllosilicates, in particular serpentine with a broad $10 \mu$ m peak. For peak pressures between $\sim 20$ and $30 \mathrm{GPa}$, the Murchison samples retain mid-IR signatures of phyllosilicate species but crystalline olivine's peak at $\sim 11.2 \mu \mathrm{m}$ begins to emerge. At higher pressures $(\sim 36 \mathrm{GPa})$ corresponding to high-energy collisions, the phyllosilicate signatures are lost due to serpentine decomposition and devolatization and the spectra better resemble those for debris disks with weaker/absent phyllosilicate emission such as HD 113766A and HD 69830. Thus, EF Cha's debris disk plausibly represents a particularly rare laboratory for studying phyllosilicates in other planetary systems, where collisions are sufficient to produce detectable debris but not so catastrophic as to eliminate evidence for aqueously altered planetesimals.

While numerous protoplanetary disks in nearby star-forming regions have been targeted by Spitzer, studies typically do not incorporate phyllosilicate species in their spectral modeling and instead focus on amorphous silicates, forsterite, enstatite, and silica (e.g., Sargent et al. 2009; Watson 2009; Juhasz et al. 2010). ${ }^{6}$ Recent models for protoplanetary disk spectra by Morris $\&$ Desch (2009) argue that phyllosilicates can be identified at $\sim 10 \mu \mathrm{m}$ and, especially, $20-25 \mu \mathrm{m}$ if they comprise more than $3 \%$ of the dust by surface area, as is the case for EF Cha, and if the dust is fine enough to be optically thin. ${ }^{7}$

However, our analysis cannot yet constrain the frequency or duration of phyllosilicate production in debris disks. No other debris disk has been reported to date as having strong, clearly phyllosilicate derived emission features. Further, since debris disk grains are generally too large to produce clear solid-state features anyway, we cannot conclude that phyllosilicate production in debris disks is fleeting or rare. More precisely, determining exactly when and how often phyllosilicates form, and thus when water is delivered to the terrestrial zone of young planetary systems, will require modeling more protoplanetary disks and debris disks with solid-state features using sophisticated mineralogical models like those presented here. We are encouraged that future work will produce more and more phyllosilicate detections, however, given the preponderance of likely phyllosilicate-rich C- and D-type asteroids in the outer main

\footnotetext{
6 Some of these studies identify solid-state features in many protoplanetary disks at wavelengths qualitatively similar to phyllosilicate features. However, these authors argue that the features are due to allotropes of silica (e.g., Sargent et al. 2009; Juhasz et al. 2010), which we have been able to rule out for EF Cha. 7 Their modeling formalism differs from ours in that they adopt a "distribution of hollow spheres" to compute dust opacities. We use laboratory emission spectra from fine powders. However, their computed emissivities qualitatively capture basic features of those for powders, specifically a strong, narrow peak at $10 \mu \mathrm{m}$.
} 
belt of the solar system, and the suggestion of phyllosilicates in comets (Lisse et al. 2006, 2007a; Reach et al. 2009a).

Spectral modeling of protoplanetary disks is also arguably more complicated, given the effects of disk flaring/shadowing, variability, and the inability to probe the disk midplane (where planetesimals should be located) if the disk is optically thick. However, many Herbig AeBe stars with protoplanetary disks in the Juhasz et al. study (HD 36112, HD 37258, HD 37357 , etc.) - the likely evolutionary precursors to older A stars with debris disks like EF Cha-have high signal-to-noise Spitzer spectra and numerous solid-state features in the $10-25 \mu \mathrm{m}$ range. While many (most?) of their disks can be fit by typical olivine+pyroxene+silica porous Mie spectral models and PAH empirical residual templates (e.g., Juhasz et al. 2010), they also provide an interesting future sample to apply our spectral modeling to search for phyllosilicate-rich protoplanetary disks.

\section{CONCLUSION}

This paper presents analysis of Spitzer IRS data for the debris disk surrounding the $10 \mathrm{Myr}$ old star, EF Cha. To investigate the composition of the EF Cha debris disk, location of the debris dust with respect to the star, and size of the emitting grains, we have compared our disk spectra to that for disks with a wide range of evolutionary stages and analyzed it using a sophisticated mineralogical model. Our study yields the following main results.

1. Using simple energy balance considerations, the EF Cha disk must be a gas-poor debris disk with LTE dust temperatures between $225 \mathrm{~K}$ and $430 \mathrm{~K}$ and dust physically located at $\sim 1-7.5$ AU. Because these temperatures are characteristic of equilibrium dust temperatures between 0.45 and 1.55 AU of the Sun in our solar system, we expect that the EF Cha debris disk contains terrestrial-zone material.

2. Unlike most debris disks, the EF Cha disk shows evidence for numerous solid-state features diagnostic of the composition of parent bodies producing its debris, implying consistent presence of small, $\mu \mathrm{m}$-sized grains around the star. Comparing the EF Cha spectrum with that for other disks exhibiting solid-state features (mostly protoplanetary disks) shows that the EF Cha disk lacks evidence for PAH emission and has relatively little crystalline olivine and pyroxene. While the general shape of the EF Cha spectrum is consistent with a high abundance of amorphous silicates, numerous maxima secondary features show that additional species must be abundantly present.

3. Mineralogical modeling shows that the EF Cha debris disk includes an exceptionally high-mass fraction of phyllosilicates, produced by aqueous alteration of anhydrous rock, making it unique among all debris disks studied thus far. Its elevated olivine to pyroxene ratio is further evidence of large-scale aqueous alteration. The dust may also contain water ice and metal sulfides.

4. The amount of dust present is the equivalent of a large, $\sim 110 \mathrm{~km}$ radius asteroid, implying the participation of a large parent body or bodies sourcing the observed dust; this sourcing could have been caused by collisions in an asteroid belt.

5. Because only a narrow range of collisional energies will yield detectable debris but not convert phyllosilicates back into silicates via devolatilization, EF Cha's debris disk represents a potentially rare laboratory for studying phyllosil- icate species - a signpost of terrestrial water delivery-in a debris disk and motivates future studies to identify these species in protoplanetary disks.

We thank Marc Kuchner, Aki Roberge, Scott Kenyon, Alycia Weinberger, John Debes, Steve Desch, Melissa Morris, and the anonymous referee for useful comments and discussions that improved the quality of this work. This paper was based on observations taken with the NASA Spitzer Space Telescope, operated by JPL/CalTech. T.C. is funded by a NASA Postdoctoral Fellowship; C.M.L. gratefully acknowledges support for performing the modeling described herein from JPL contract 1274485, NSF AAG grant NNX09AU31G-1, and the APL Janney Fellowship program. A.S.-A. acknowledges support from the Deutsche Forschungsgemeinschaft (DFG) grant SI 1486/ 1-1. K.Y.L.S. and G.R. were partially supported by contract 1255094 from Caltech/JPL to the University of Arizona.

\section{REFERENCES}

A'Hearn, M. F., et al. 2005, Science, 310, 258

Akai, J. 1992, Antarct. Meteorol. Res., 5, 120

Backman, D., \& Paresce, F. 1993, in Protostars and Planets III, ed. E. H. Levy \& J. I. Lunine (Tucson, AZ: Univ. Arizona Press), 1253

Bell, J. F., et al. 1989, in Asteroids II, ed. R. P. Binzel, T. Gehrels, \& M. S. Matthews (Tucson, AZ: Univ. Arizona Press), 921

Bibring, J.-P., et al. 2006, Science, 312, 400

Bitner, M., et al. 2007, ApJ, 661, L69

Bouwman, J., et al. 2001, A\&A, 375, 950

Bouwman, J., et al. 2008, ApJ, 683, 479

Bradley, J., et al. 1999, Science, 285, 1716

Bus, S., \& Binzel, R. 2002, Icarus, 158, 106

Campins, H., et al. 2010, Nature, 464, 1320

Carmona, A., et al. 2008, A\&A, 477, 839

Chen, C. H., et al. 2006, ApJS, 166, 351

Chihara, H., et al. 2002, A\&A, 391, 267

Ciesla, F., \& Lauretta, D. 2005, Earth Planet. Sci. Lett., 231, 1

Currie, T., \& Kenyon, S. J. 2009, AJ, 138, 703

Currie, T., et al. 2007a, ApJ, 659, 599

Currie, T., et al. 2007b, ApJ, 663, L105

Currie, T., et al. 2007c, ApJ, 669, L33

Currie, T., et al. 2008a, ApJ, 672, 558

Currie, T., et al. 2008b, ApJ, 688, 597

Currie, T., et al. 2009, ApJ, 698, 1

Currie, T., et al. 2010, ApJS, 186, 191

Dahm, S., \& Carpenter, J. 2009, AJ, 137, 4024

Desch, S. J., \& Leshin, L. A. 2004, Proc. 35th Lunar Planet. Sci. Conf., Abstract 1987

Farmer, J. D., \& Des Marais, D. J. 1999, J. Geophys. Res., 104, 26977

Fegley, B., \& Prinn, R. 1989, in The Formation and Evolution of Planetary Systems, ed. H. A. Weaver \& L. Danley (Cambridge: Cambridge Univ. Press), 171

Ford, E., \& Chiang, E. 2007, ApJ, 661, 602

Goldreich, P., Lithwick, Y., \& Saari, R. 2004, ARA\&A, 42, 549

Golimowski, E., et al. 2006, AJ, 131, 3109

Gorlova, N., et al. 2007, ApJ, 617, 560

Gorti, U., \& Hollenbach, D. 2004, ApJ, 613, 424

Gradie, J., \& Tedesco, E. 1982, Science, 216, 1405

Grady, C., et al. 2005, ApJ, 630, 958

Hanner, M. S., \& Zolensky, M. E. 2010, in Astromineralogy, ed. T. Henning (2nd ed.; Berlin: Springer), 203

Hernandez, J., et al. 2009, ApJ, 707, 705

Higdon, S., et al. 2004, PASP, 116, 975

Juhasz, A., et al. 2010, ApJ, 721, 431

Jura, M. 2006, ApJ, 653, 613

Jura, M., et al. 2009, ApJ, 699, 1473

Kennedy, G., \& Wyatt, M. 2010, MNRAS, 405, 1253

Kenyon, S. J., \& Bromley, B. 2004, ApJ, 602, L133

Kenyon, S. J., \& Bromley, B. 2008, ApJS, 179, 451

Kenyon, S. J., \& Bromley, B. 2010, ApJS, 188, 242

Koike, C. 2000, A\&A, 363, 1115

Koike, C., et al. 2002, A\&A, 399, 1101

Krivov, A. 2010, Res. Astron. Astrophys., 10, 383

Kuchner, M. 2003, ApJ, 596, L105 
Lagrange, A.-M., et al. 2010, Science, 329, 57

Lisse, C., et al. 2006, Science, 313, 635

Lisse, C., et al. 2007a, Icarus, 187, 69

Lisse, C., et al. 2007b, ApJ, 658, 584

Lisse, C., et al. 2008, ApJ, 673, 1106

Lisse, C., et al. 2009, ApJ, 701, 2019

Lisse, C., et al. 2011, submitted

Maness, H., et al. 2008, ApJ, 686, L25

Martin-Zaidi, C., et al. 2010, A\&A, 516, 110

Matsumura, S., et al. 2010, ApJ, 714, 194

Meeus, G., et al. 2001, A\&A, 365, 476

Merk, R., \& Prialnik, D. 2006, Icarus, 183, 283

Morales, F. Y., et al. 2009, ApJ, 699, 1067

Morlok, A., et al. 2010, Icarus, 207, 45

Morris, M., \& Desch, S. J. 2009, Astrobiology, 9, 965

Mouillet, D., et al. 1997, MNRAS, 292, 896

Mustill, A., \& Wyatt, M. C. 2009, MNRAS, 399, 1403

Nagahara, H., \& Ozawa, K. 2011, Proc. 42nd Lunar Planet. Sci. Conf., Abstract 2838

Orofino, V., et al. 2010, Icarus, 208, 202

Pascucci, I., et al. 2006, ApJ, 651, 1177

Pascucci, I., et al. 2007, ApJ, 663, 383
Reach, W. T., et al. 2009a, ApJ, 690, 683

Reach, W. T., et al. 2009b, ApJ, 693, 697

Rhee, J., et al. 2007, ApJ, 671, 616

Rieke, G. H., et al. 2005, ApJ, 620, 1010

Rivkin, A. S., \& Emery, J. P. 2010, Nature, 464, 1322

Robert, F., et al. 2000, Space Sci. Rev., 92, 201

Sargent, B., et al. 2009, ApJS, 182, 477

Schneider, G., et al. 2009, ApJ, 137, 53

Sitko, M., et al. 2008, ApJ, 678, 1070

Skrutskie, M., et al. 2006, AJ, 131, 1163

Stansberry, J., et al. 2004, ApJS, 154, 463

Swain, M., et al. 2008, ApJ, 674, 482

Tomeoka, K. 1990, Nature, 345, 138

Tomeoka, K., \& Ohnishi, I. 2010, Geochim. Cosmochim. Acta, 74, 4438

Vilas, F., \& Gaffey, M. J. 1989, Science, 246, 790

Wahhaj, A., et al. 2007, ApJ, 661, 368

Wasson, J. 2008, Icarus, 195, 895

Watson, D. 2009, in ASP Conf. Ser. 414, Cosmic Dust-Near and Far, ed. Th. Henning, E. Grun, \& J. Steinacker (San Francisco, CA: ASP), 77

Wyatt, M. C. 2008, ARA\&A, 46, 339

Wyatt, M. C., et al. 2005, ApJ, 620, 492

Wyatt, M. C., et al. 2007, ApJ, 663, 365 